\title{
Surprising evolutionary predictions from enhanced ecological realism
}

\author{
Ulf Dieckmann ${ }^{\mathrm{a}, \mathrm{b}, *}$, Johan A.J. Metz ${ }^{\mathrm{b}, \mathrm{a}}$ \\ ${ }^{a}$ Evolution and Ecology Program, International Institute for Applied Systems Analysis, Schlossplatz 1, A-2361 Laxenburg, Austria \\ ${ }^{\mathrm{b}}$ Section Theoretical Biology, Institute of Biology, Leiden University, Kaiserstraat 63, NL-2311 GP Leiden, The Netherlands
}

Received 8 August 2005

Available online 15 February 2006

\begin{abstract}
A focus on the eco-evolutionary feedback continually operating between a population's evolution and its environment helps to appreciate the generality of ESS theory. Here we illustrate, through a sequence of four examples, how respecting such feedback in the evolutionary dynamics of quantitative traits may result in qualitatively unexpected outcomes. Reviewing existing insights and complementing these with new results, we show (1) that evolutionary matrix games are fundamentally degenerate and allow a natural unfolding, (2) that selection-driven extinction may not be rare in nature, (3) that evolutionary epidemiology should not rely on $R_{0}$ maximization, and (4) why the occurrence of Hardy-Weinberg proportions generically requires an evolutionary explanation.

(C) 2005 Elsevier Inc. All rights reserved.
\end{abstract}

Keywords: Eco-evolutionary feedback; Matrix games; Unfolding evolutionarily singular points; Evolutionary suicide; Evolutionary epidemiology; Optimization principles; Deviations from Hardy-Weinberg; Sex-dependent fitness; Continuous stability

\section{Introduction}

ESS theory provides the link between evolutionary mechanisms and natural history. Its strength lies in asking and answering deep questions about readily observable biological phenomena, be they ecological, behavioral, physiological, or morphological. ESS theory does so by concentrating on the phenotype and its role in the interaction among organisms, while neglecting genetic detail. It is through this simplification and focus that ESS theory facilitates a fruitful interplay between evolutionary modeling and experimental and observational biology.

ESS theory originally arose from attempts to understand the evolutionary underpinnings of behavioral phenotypes. In such contexts, the need to account for the phenotypes of other individuals that a focal individual will interact with was particularly evident (Maynard Smith and Price, 1973; Maynard Smith, 1982). In this way, ESS theory helped emphasize the importance of frequency-dependent selec-

\footnotetext{
*Corresponding author. Evolution and Ecology Program, International Institute for Applied Systems Analysis, Schlossplatz 1, A-2361 Laxenburg, Austria. Fax: + 43223671313 .

E-mail address: dieckmann@iiasa.ac.at (U. Dieckmann).
}

tion for understanding natural evolutionary change: in models for the evolution of behavior, the fitness of individuals is bound to depend on a population's phenotypic composition.

Approaching the feedback between a population's composition and the underlying selection pressures from a more population dynamical angle, research in the 1970s also made important progress in the analysis of evolutionary outcomes under density-dependent selection (Roughgarden, 1971, 1979). As it turned out, the method of predicting evolutionary outcomes by optimizing a suitably chosen fitness function could be extended from problems with density- and frequency-independent selection to those with purely density-dependent selection. In the former case, all fitness values are constant over time, while in the latter case they vary with (and only with) a population's density.

For almost two decades, these alternative approaches remained curiously disparate. Students of evolution could either rely on optimization methods, if they felt they could safely ignore frequency-dependent selection, or on methods broadly referred to as "game theoretical," if they had a hunch that frequency dependence was germane to the evolutionary question at hand. This state of affairs, 
however, was rather unsatisfactory: not only did it prevent the transparent and accurate analysis of evolutionary problems in which density- and frequency-dependent selection both played a role, but it also fostered the hopeful belief that most problems in life-history evolution could be solved through optimization methods, if only suitable fitness functions could be identified (e.g., Stearns, 1992; Roff, 1992; for a counterexample, see Heino et al., 1997).

The impasse in bringing together models of evolution under density- and frequency-dependent selection was overcome gradually. Two key ideas played a role in this process. The first idea, already foreshadowed in the context of game theoretical and optimization methods, is to envisage fitness always as a function of both phenotype and environment. To fully appreciate this point and make it operational, a suitable formal definition of 'environment' had to be established. Since the environment experienced by an individual is bound to depend on the current state of its population, this environment may be affected, first, by the population's phenotypic composition, and second, for an unstructured population, by the population's total abundance. The first dependence applies whenever selection is frequency-dependent, and the second one whenever selection is density-dependent. (In structured populations, the neat divide between the two types of selection becomes blurred whenever densities of life stages affect phenotypes differentially. For further elaborations of the concept of frequency-dependent selection in the spirit of the present article, see Heino et al., 1998.) In general, the evolutionary environment of an individual is to be described such that all outside influences impinging on any individuals contributing to the dynamics of the focal population, now and in the future, are covered. The evolutionary environment thus defined differs from the instantaneous environment familiar from describing population dynamics. For the latter, only the current influences on the individuals in the focal population are considered. Both formal notions of environment have been inspired by the theory of physiologically structured populations (Metz and de Roos, 1992; Metz and Diekmann, 1986; Diekmann et al., 2001, 2003). The second idea facilitating a merger of game theoretical and optimization methods is to derive the fitness functions to be analyzed from an individual-based view of the underlying population dynamics - in earlier work, such functions had often been instead assumed a priori. Taken together, these ideas motivated the introduction of the so-called invasion fitness of a given phenotype in a given environment, defined and derived - analytically, numerically, or empirically — as the phenotype's asymptotic exponential growth rate in the considered environment (Metz et al., 1992; Rand et al., 1994).

The characteristic idea of ESS theory is that the phenotypes currently present in a community are continually challenged by variants appearing in such small numbers that they do not perceivably perturb the community's attractor. An ESS is a phenotype, or set thereof, that cannot be invaded by any such rare variants. Invasion fitness helps to predict the dynamics of invasion attempts. In an ecologically stationary community, the invasion fitness of all present phenotypes equals zero by definition (otherwise, the community would not be stationary). By contrast, rare phenotypes with positive invasion fitness may invade (usually after many unsuccessful trials, owing to demographic stochasticity in the invader population), while those with negative invasion fitness cannot invade (unless the invaded population as a whole is so small that it is subject to considerable demographic stochasticity). In principle, the fate of variants with zero invasion fitness would have to be determined from higher-order terms however, as we shall argue in Section 2, the case of variants that possess zero invasion fitness and are not equal to a present phenotype is so non-generic that it can safely be neglected for most intents and purposes.

Nothing said so far is overly new. Not only that, there are many earlier studies that have implicitly shared these ideas and have contributed to their practical development. Yet it seems that the unifying potential resulting from the perspective established above remains underexploited. The key notion here is that of the eco-evolutionary feedback loop, continually operating between a population's evolution and its environment: while the environment determines current selection pressures, these pressures change a community's phenotypic composition, which in turn alters its environment. Under such feedback, fitness landscapes are varying in shape as evolutionary changes unfold. By explicitly introducing this feedback loop, based on a suitable formal notion of environment, many disparate evolutionary investigations and phenomena can be brought under one heading, thus unifying the analysis of large classes of model families. Perhaps even more importantly, the evolutionary properties of realistically complicated models are often becoming more accessible through this natural conceptual decomposition of the ecological theatre.

ESS theory today has clearly moved beyond earlier models based on ad hoc payoff matrices or unproven optimization principles. In this article we tout the idea that the full generality of ESS theory can best be brought out by concepts and tools designed to analyze eco-evolutionary feedback. By firmly rooting evolutionary predictions in the underlying population dynamics, fairly realistic ecological scenarios can be tackled. Below, we will highlight a range of evolutionary surprises resulting from such enhanced levels of ecological realism. The examples presented in the following four sections are also meant to illustrate some of the technical principles that, in our opinion, underlie the generalization of ESS theory.

\section{The fundamental degeneracy of matrix games can be unfolded}

The straightforward tractability of matrix games and ESS conditions has enabled game theory to become an important 
and popular framework for modeling phenotypic evolution. This success, however, has come at a price, for two reasons:

- First, beyond stylized games of behavior, continuous strategies usually offer a more convincing rendering of real evolving traits than discrete strategies. By contrast, in game theoretical models of biological evolution, continuous strategies are typically introduced and analyzed merely as mixtures of pure strategies.

- Second, for many questions in evolutionary ecology it is necessary to consider the full population dynamical effects of strategies. In models based on matrix games, an attempt is made, instead, to infer the effects of strategies on fitness directly from payoff matrices. Such an approach also complicates the integration of realistic types of density regulation into models based on matrix games.

Whenever applications of evolutionary game theory consider mixed strategies in matrix games, a peculiar degeneracy raises its ugly head. This degeneracy directly follows from how matrix-game payoffs of mixed strategies are determined. Let us consider a resident population with mixed strategy $x$. Here, the components of the vector $x$ describe the probabilities $x_{i}$ with which the resident players follow any one of $k$ pure strategies $i=1, \ldots, k$, with $\sum_{i=1}^{k} x_{i}=1$. The components $W_{i j}$ of the payoff matrix $W$ describe the payoff received by a player adopting the pure strategy $i$ against a player using the pure strategy $j$. The average payoff of players with a rare variant strategy $x_{\mathrm{v}}$ is $x_{\mathrm{v}}^{T} W x$ (where $T$ denotes transposition), so that their excess payoff relative to a resident player is $f\left(x_{\mathrm{v}}, x\right)=x_{\mathrm{v}}^{T} W x-$ $x^{T} W x$. Since it is assumed that variants with $f>0$ can invade, while those with $f<0$ cannot, the sign of $f$ carries the same information as that of invasion fitness in models with explicit population dynamics. It is already clear from this observation that the game-theoretical case is rather special: whereas invasion fitness functions may be (and usually are) nonlinear, mixed strategies in matrix games inevitably lead to functions $f$ that are linear in both $x$ and $x_{\mathrm{v}}$.

It is instructive to explore the consequences of this degeneracy by considering games with just two pure strategies. For $k=2$, a single adaptive trait suffices, $x=$ $(p, 1-p)$ and $x_{\mathrm{v}}=\left(p_{\mathrm{v}}, 1-p_{\mathrm{v}}\right)$,

$$
\begin{aligned}
f\left(p_{\mathrm{v}}, p\right)= & {\left[p_{\mathrm{v}} p W_{11}+p_{\mathrm{v}}(1-p) W_{12}\right.} \\
& \left.+\left(1-p_{\mathrm{v}}\right) p W_{21}+\left(1-p_{\mathrm{v}}\right)(1-p) W_{22}\right] \\
& -\left[p^{2} W_{11}+p(1-p) W_{12}+(1-p) p W_{21}\right. \\
& \left.+(1-p)^{2} W_{22}\right]=\left(p_{\mathrm{v}}-p\right)\left[p\left(W_{11}-W_{21}\right)\right. \\
& \left.+(1-p)\left(W_{12}-W_{22}\right)\right] .
\end{aligned}
$$

From this we can see that $f\left(p_{\mathrm{v}}, p\right)=0$ for $p_{\mathrm{v}}=p$ and for $p=p^{*}$ with $p^{*}=\left(W_{22}-W_{12}\right) /\left(W_{11}-W_{12}+W_{22}-\right.$ $\left.W_{21}\right)$. Fig. 1a illustrates the resultant pairwise invasibility plot for $0<p^{*}<1$. This plot highlights two geometric
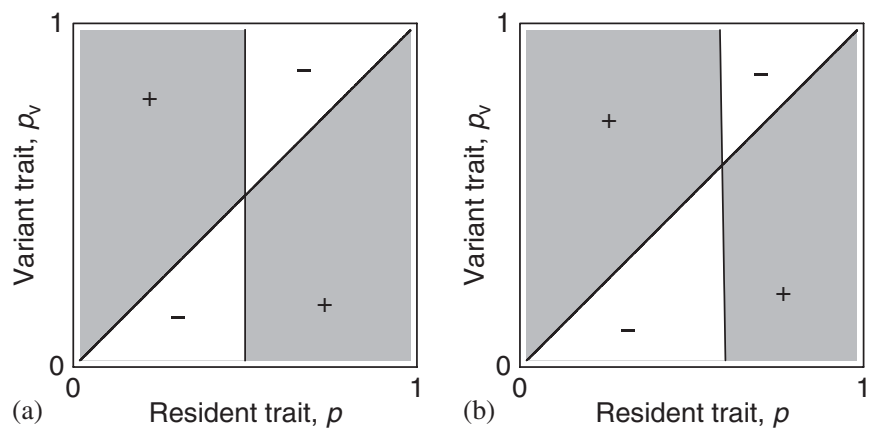

Fig. 1. Pairwise invasibility plots for (a) the original hawk-dove game and for (b) an ecological embedding with fluctuating rewards. The nondiagonal zero-contour curve is strictly straight and vertical in (a), whereas this curve is tilted counter-clockwise and concave from the right in (b). Even though both effects are only slight, they do imply a qualitative change in evolutionary behavior. Parameters: $V=0.5, C=1, R=2$, and $c=0$ (a), $c=1$ (b).

consequences of the linearity of $f$ : the non-diagonal zerocontour curve of $f$ at $p=p^{*}$ is both straight and vertical. In other words, in a population of players following the mixed strategy $p^{*}$, all variant strategies are strictly neutral, $f\left(p_{\mathrm{v}}, p^{*}\right)=0$ for all $p_{\mathrm{v}}$. This neutrality extends to the effect that all mixtures of mixed and/or pure strategies resulting in a population with strategy $p^{*}$ are evolutionarily neutral as well. These observations reflect a general result of game theory, widely known as the Bishop-Cannings theorem (Bishop and Cannings, 1978). It is thus immediately clear that, when viewed in a broader context, the treatment of mixed strategies in matrix games is structurally unstable: the slightest variation in model structure is likely to destroy the degenerate geometry depicted in Fig. 1a, by bending and/or tilting the non-diagonal zero-contour curve away from a straight and vertical line. At the same time, these variations remove the evolutionary neutrality of mixtures with population strategy $p^{*}$.

Below we show how the described degeneracy is readily overcome when invasion fitness is derived from population dynamics into which realistic aspects of ecological interactions are incorporated. To keep these discussions as concrete as possible, we focus on the classical hawk-dove game for illustration (Maynard Smith, 1982). In this game, players adopt interaction strategies that are either selfish (hawk) or cooperative (dove). When a hawk plays against a dove, the hawk gains a reward of value $V \geqslant 0$, while the dove gains nothing. When two doves play against one another, they share the reward, each gaining $\frac{1}{2} V$. By contrast, when two hawks interact, both of them gain $\frac{1}{2}(V-C)$ on average, where $C \geqslant 0$ measures the cost of hawkish encounters. With $p$ and $p_{\mathrm{v}}$ denoting the probabilities with which the hawk strategy is used by resident and variant players, respectively, the rare variant's excess payoff is given by

$f\left(p_{\mathrm{v}}, p\right)=\frac{1}{2}\left(p_{\mathrm{v}}-p\right)(V-p C)$,

implying $p^{*}=V / C$. Thus, whenever the cost $C$ exceeds the reward $V$, evolution is expected to converge on the mixed 
strategy $p^{*}$ (for $C \leqslant V$, evolution will instead increase $p$ up to $p=1)$. Again, once $p^{*}$ is resident, all variants are neutral.

We now examine three slight variations of the classical hawk-dove game. First, we consider a simple population dynamical embedding of the game; second, we relax the assumption that rewards are fixed; and third, we relax the assumption of fixed interaction rates. For simplicity, we consider discrete-time models with non-overlapping generations. We assume that each individual has an intrinsic reproduction ratio $R \geqslant 0$, which is enhanced by payoffs from the hawk-dove game and diminished by density regulation. For a rare variant strategy $p_{\mathrm{v}}$ in a resident population with strategy $p$ at population dynamical equilibrium, this results in a reproduction ratio of $\left\{R+\frac{1}{2} r\left[V\left(1-p+p_{\mathrm{v}}\right)-C p p_{\mathrm{v}}\right]\right\} / F(p)$, where $r \geqslant 0$ is the per generation rate at which individuals interact by engaging in the hawk-dove game. The density-regulating factor $F$ is obtained from observing that the resident's own reproduction ratio equals 1 at population dynamical equilibrium, which gives $F(p)=R+\frac{1}{2} r\left[V-C p^{2}\right]$. Notice that the density dependence thus considered is selectively neutral, in that it affects all phenotypes alike. Consequently, the hawk-dove game's degeneracy is preserved in this simple population dynamical embedding. Based on these assumptions, we obtain the model's invasion fitness as the logarithm of the variant's reproduction ratio,

$f\left(p_{\mathrm{v}}, p\right)=\ln \frac{R+\frac{1}{2} r\left[V\left(1-p+p_{\mathrm{v}}\right)-C p p_{\mathrm{v}}\right]}{R+\frac{1}{2} r\left[V-C p^{2}\right]}$.

It is straightforward to verify that (3) is sign-equivalent to (2).

As a further variation, we now relax the assumption that rewards in the hawk-dove game are strictly fixed. In realistic ecological settings, it is likely that such rewards are fluctuating between generations, reflecting, for example, variations in environmental conditions between years. To keep the treatment transparent, we consider the simplest such fluctuation by assuming that the reward can switch between just two values, $V_{1}=(1+c) V$ and $V_{2}=(1+c)^{-1} V$, where $c \geqslant 0$ measures the contrast between these two rewards. In each generation, values $V_{1}$ and $V_{2}$ are attained with equal probability. Whereas this perturbation of the original model thus leaves the geometric mean of the reward invariant, the model's invasion fitness, obtained from the geometric mean of the reproduction ratio resulting for the two reward values, is changed to

$f\left(p_{\mathrm{v}}, p\right)=\frac{1}{2} \ln \prod_{i=1}^{2} \frac{R+\frac{1}{2} r\left[V_{i}\left(1-p+p_{\mathrm{v}}\right)-C p p_{\mathrm{v}}\right]}{R+\frac{1}{2} r\left[V_{i}-C p^{2}\right]}$.

Fig. 1b illustrates a resultant pairwise invasibility plot. It turns out that the slightest reward contrast removes the formerly observed degeneracy. Specifically, for any $c>0$, the slope of the non-diagonal zero-contour curve of $f$ is negative (i.e., the curve is tilted counter-clockwise), while its curvature is positive (i.e., the curve is concave from the right). Accordingly, the previously existing plethora of evolutionarily neutral mixtures has collapsed to a unique monomorphic attractor. This delicate sensitivity underscores the structural instability of the original model.

So far, we have assumed that the rate $r$ at which individuals interact through the hawk-dove game is strictly identical for all players. However, in ecologically realistic circumstances, it is quite likely that players are subtly or significantly more or less likely to engage in such interactions, depending on their strategy. Doves may avoid interactions and hawks may seek out engagements, or vice versa. To explore the consequences of such variation with some generality, we expand the interaction rates for strategies $p$ up to second order around $p^{*}$,

$r(p)=r_{0}+\left(p-p^{*}\right) r_{1}+\left(p-p^{*}\right)^{2} r_{2}$

with $r_{0} \geqslant 0$, and update the model's invasion fitness accordingly,

$f\left(p_{\mathrm{v}}, p\right)=\ln \frac{R+\frac{1}{2} \tilde{r}\left(p_{\mathrm{v}}, p\right)\left[V\left(1-p+p_{\mathrm{v}}\right)-C p p_{\mathrm{v}}\right]}{R+\frac{1}{2} r(p)\left[V-C p^{2}\right]}$.

Here the choice of $\tilde{r}\left(p_{\mathrm{v}}, p\right)=\sqrt{r\left(p_{\mathrm{v}}\right) r(p)}$ reflects the assumption that engagements are initiated bilaterally and symmetrically. Fig. 2 illustrates the resultant pairwise invasibility plots. As can be seen, the slightest departure from uniform interaction rates removes the game-theoretical degeneracy. Notice that in this third variant of the model the non-diagonal zero-contour curve of $f$ can have either positive or negative slope, and also its curvature can either be positive or negative.

More in general, the third model variant shows how the degenerate game-theoretical case serves as the organizing center (Golubitsky and Schaeffer, 1985) of a rich bifurcation structure. Since the signs of both slope and curvature of the non-diagonal zero-contour curve of invasion fitness qualitatively affect evolutionary predictions, the local unfolding of the game-theoretical degeneracy always requires variation of at least two model parameters. Using the non-diagonal zero-contour curve's slope and curvature at $p=p^{*}$ as generic local unfolding parameters, the degeneracy's unfolding can be depicted as shown in Fig. 3. Notice that, in this unfolding, transitions from negative to positive slopes are of particular relevance, since these correspond to the transformation of an evolutionarily stable strategy into an evolutionary branching point (Metz et al., 1996a; Geritz et al., 1997). Consequently, expected evolutionary outcomes fundamentally differ on either side of such a transition: for an evolutionarily stable strategy, a monomorphic mixed strategy is expected to evolve, whereas evolutionary branching points may give rise to population-level dimorphisms of strategies.

Interestingly, the members of the dimorphisms eventually emerging after evolutionary branching may both be either pure or mixed strategies. A full analysis distinguishing the various cases then enables a much more conclusive 


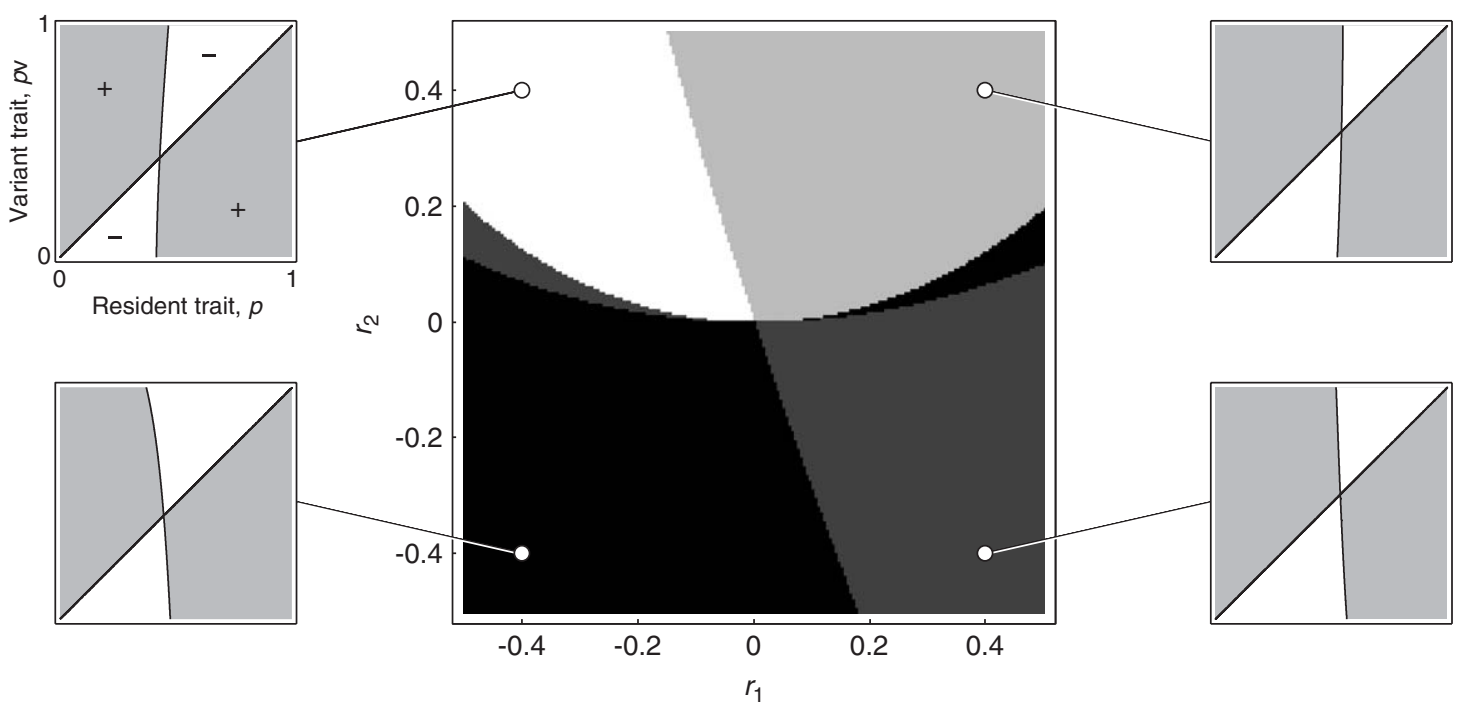

Fig. 2. Pairwise invasibility plots and unfolding for an ecological embedding of the hawk-dove game with variable interaction rates. The classical gametheoretical case (Fig. 1a) is located at $r_{1}=r_{2}=0$; it is thus straddling three bifurcation curves, reflecting its structural instability. In the top-left panel, the non-diagonal zero-contour curve is tilted clockwise and is concave from the right; in the top-right panel, the curve is titled clockwise and is concave from the left; in the bottom-left panel, the curve is tilted counter-clockwise and is concave from the left; and in the bottom-right panel, the curve is tilted counterclockwise and is concave from the right. Evolutionary branching is predicted to occur for parameters in the white and light gray regions of the central panel, as can be seen from the top-left and top-right pairwise invasibility plots. Parameters: $V=0.5, C=1, R=2$, and $r_{0}=1$.

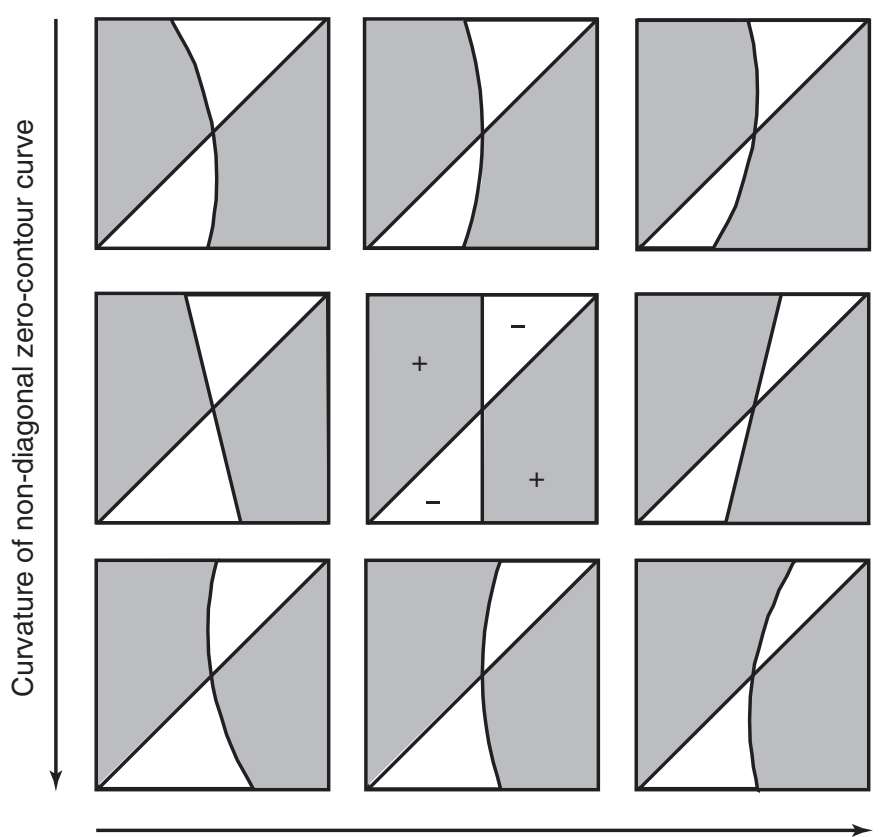

Slope of non-diagonal zero-contour curve

Fig. 3. General unfolding of mixed ESS in evolutionary matrix games.

prediction of the eventual evolutionary outcomes than is possible based on matrix games. Unfolding the degeneracy of mixed strategies in matrix games thus results not only in the removal of a perilous structural instability, but also offers an additional conceptual benefit: whereas, in matrix games, mixed strategies realized probabilistically at the level of individuals or polymorphically at the level of populations are indistinguishable, instructive insights into the interplay between these biologically rather different realizations of diversity can be gained once the gametheoretical degeneracy is overcome.

In this section we have shown how a more extensive population dynamical embedding and the addition of salient elements of ecological realism help to unfold a fundamental degeneracy of evolutionary matrix games. We suggest that the only features of an evolutionary game likely to be biologically relevant are those that stay intact under such an unfolding.

\section{Selection-driven extinctions need not be rare}

For a long period during the 19th and 20th centuries, evolution was thought to operate so as to benefit the affected species. Accordingly, it was widely expected that, for example, life-history evolution would always enhance a population's viability. Such was Darwin's confidence in this prowess of adaptive evolution that he suggested "we may feel sure that any variation in the least degree injurious would be rigidly destroyed" (Darwin, 1859, p. 130) and "Natural selection will never produce in a being anything injurious to itself, for natural selection acts solely by and for the good of each" (Darwin, 1859, p. 228).

Notions of optimizing selection are underlying landmarks of evolutionary theory developed during the Modern Synthesis, like Fisher's so-called fundamental theorem of natural selection (Fisher, 1930), or Wright's notion of hill climbing on genotypically or phenotypically defined fitness landscapes (Wright, 1932, 1967). Also Levins's fitness-set approach to the study of bivariate evolution (Levins, 1962, 1968), still enjoying widespread recognition in lifehistory evolution (Yodzis, 1989, pp. 324-351; Case, 1999, 
pp. 175-177; Calow, 1999, p. 758), is based on the assumption that, within a set of feasible phenotypes defined by a trade-off, evolution will maximize a population's fitness.

The perception that evolution worked for the good of the species was also common among field biologists, often based on implicit or explicit ideas of group selection, which found their culmination in the work by Wynne-Edwards (1962). By the 1970s, explaining adaptations in terms of species-level benefits had fallen into disrepute (Williams, 1966). While most biologists are thus aware that adaptive evolution, in principle, can undermine a population's viability, and while such phenomena are regularly discussed in the context of the 'tragedy of the commons' (Hardin, 1968), the evolution of altruism (e.g., Axelrod and Hamilton, 1981), or the evolution of sex (e.g., Maynard Smith, 1978), the role of adaptive life-history evolution in causing extinctions has received but limited attention to date.

A notion still lingers in the biological community that it should be only under very exceptional circumstances that adaptive evolution worsens a population's lot to the extent of causing extinction. Earlier ESS theory (e.g., Maynard Smith, 1982) did not address this issue, since classical matrix games are not concerned with the impact of strategies on population density. Also modern applications of ESS theory based on the replicator equation (Taylor and Jonker, 1978; Schuster and Sigmund, 1983; Hofbauer and Sigmund, 1998) tend to focus attention on changes in the frequencies, rather than the density, of strategies. For an alternative approach to game dynamics that aimed at including densities, see Cressman (1990).

While classical ESS theory, then, does not easily lend itself to the study of selection-driven extinction, frequencydependent selection, a consistently emphasized aspect of ESS theory, plays an important role in such processes. Frequency-dependent selection is crucial for understanding selection-driven extinction because it allows the invasion of populations by a strategy that is beneficial to individuals as long as that strategy is rare, while ruining the population's viability once that strategy has become common. Models of selection-driven extinction cannot do without densitydependent selection either: if fitness values are independent of density, equilibrium population densities, and thus extinctions, cannot be predicted. It thus becomes clear that models of selection-driven extinction need to include both frequency- and density-dependent selection. In other words, they must incorporate a sufficient degree of ecological realism.

A verbal and lucid example of a mechanism capable of causing selection-driven extinction comes from considering overtopping growth in plants. Taller trees get more sunlight while casting shade onto their neighbors. As selection causes the average tree height to increase, fecundity declines, as more of the tree's energy budget is diverted from seed production to wood production. Under such circumstances it may also take longer for trees to reach maturity. Thus, arborescent growth as an evolutionary response to selection for competitive ability can cause deterioration both in a population's carrying capacity and in its intrinsic growth rate. The logical conclusion of such a process may be population extinction, as was first explained by Haldane (1932). Various later authors have explored eco-evolutionary models of selection-driven extinction based on similar ingredients. Below we provide a review of three salient studies.

Matsuda and Abrams (1994a) analyzed a Lotka-Volterra model in which individuals are subject to asymmetric competition and a carrying capacity that depends on their body size. Specifically, the competitive impact experienced by an individual with body size $x_{\mathrm{v}}$ in a population with mean body size $\bar{x}$ was assumed to be $\alpha\left(x_{\mathrm{v}}, \bar{x}\right)=$ $\exp \left(-h_{\alpha}\left(x_{\mathrm{v}}-\bar{x}\right)\right)$, and the carrying capacity of a population monomorphic in body size $x_{\mathrm{v}}$ was $K(x)=$ $K_{0} \exp \left(-h_{K}\left(x_{\mathrm{v}}\right)\right)$. The nonlinear function $h_{\alpha}$ preserved the sign of its argument, and the non-negative function $h_{K}$ went to infinity when its argument did. Matsuda and Abrams (1994a) concluded that, under these circumstances, adaptive evolution continues to increase body size indefinitelyprovided the advantage of large body size (as described by $h_{\alpha}$ ) is big enough and the cost of increased body size (as described by $h_{K}$ ) is small enough. Since large body sizes resulted in small carrying capacities, adaptive evolution thus perpetually diminished population density, a phenomenon Matsuda and Abrams (1994a) called 'runaway evolution to self-extinction'. Since population density in this model never vanished (it just continued to deteriorate), additional stochastic factors were required to explain extinction. Mathias and Kisdi (in press) modeled such extinctions explicitly.

In a model by Dercole et al. (2002), the per capita growth rate in a monomorphic population with adult body size $x$ and population density $N(x)$ has a logistic component $r(x)-\alpha(0) N(x)$, with the monotonically decreasing function $r(x)$ capturing the negative influence of larger body size on fecundity, and with $\alpha(0) N(x)$ measuring the extra mortality caused by intraspecific competition between individuals of the same body size. As in the previous model, the function $\alpha$ measured the competitive impact between individuals: for phenotypes $x$ and $x_{\mathrm{v}}$, the competitive impact of $x$ on $x_{\mathrm{v}}$ is $\alpha\left(x-x_{\mathrm{v}}\right) N(x)$, where $\alpha$ increases with $x-x_{\mathrm{v}}$, implying asymmetric competition. Dercole et al. (2002) also incorporated an allee effect by reducing per capita growth rates in proportion to $N(x)^{2} /\left[1+N(x)^{2}\right]$. This allee effect caused bistability in equilibrium population densities $N^{*}(x)$ : for low $x$, only a high-density equilibrium existed, for high $x$, only a lowdensity equilibrium existed, and for intermediate $x$, the two stable equilibria coexisted. The selection pressure on $x$ could be derived from the assumptions summarized here and turned out to possess two antagonistic components: the assumed shape of $r$ favored small adult body size, whereas the asymmetry of competition favored larger body size. Consequently, strong competition at the high-density 
equilibrium increased body size evolutionarily, while the dominance of the fecundity effect of body size at the lowdensity equilibrium decreased body size. The interplay of ecology and evolution in this model thus brought about an evolutionary hysteresis: body size increased at the highdensity equilibrium until the population dropped to the low-density equilibrium, at which point body size decreased until the population switched back to the highdensity equilibrium. At the low-density equilibrium, demographic or environmental stochasticity was expected to result in a greatly elevated extinction risk.

Also a model developed by Gyllenberg and Parvinen (2001) was based on asymmetric competition and the incorporation of an Allee effect. Their model is similar to the previous one, except for three features: fecundity $b(x)$ was assumed to be peaked at an intermediate value of body size $x$, a trait- and density-independent mortality $d$ was considered, and the allee effect reduced fecundity by the factor $N(x) /[1+N(x)]$. The model's invasion fitness was thus given by

$f\left(x_{\mathrm{v}}, x\right)=b\left(x_{\mathrm{v}}\right) N^{*}(x) /\left[1+N^{*}(x)\right]-d-\alpha\left(x-x_{\mathrm{v}}\right) N^{*}(x)$.

The invasion fitness yields the model's equilibrium density and selection gradient. The equilibrium density $N^{*}(x)$ is inferred from $f(x, x)=0$,

$$
\begin{aligned}
& N^{*}(x)=0, \\
& \frac{1}{2 \alpha(0)}\left\{b(x)-d-\alpha(0) \pm \sqrt{\left.[b(x)-d-\alpha(0)]^{2}-4 d \alpha(0)\right]}\right\} .
\end{aligned}
$$

The extinction equilibrium $N^{*}(x)=0$ was stable for all $x$. For intermediate values of $x$, two positive equilibria coexisted, with the high-density one being stable, separated from the extinction equilibrium by an unstable low-density equilibrium. The model's selection gradient,

$$
\begin{aligned}
g(x) & =\left.\frac{\partial}{\partial x_{\mathrm{v}}} f\left(x_{\mathrm{v}}, x\right)\right|_{x_{\mathrm{v}}=x} \\
& =b^{\prime}(x) N^{*}(x) /\left[1+N^{*}(x)\right]-\alpha^{\prime}(0) N^{*}(x),
\end{aligned}
$$

was positive for all $x$, provided that $\alpha^{\prime}(0)$ was sufficiently negative, i.e., whenever competition was sufficiently asymmetric. The adaptive dynamics of body size $x$ thus drove the population to the threshold at which the two positive equilibria vanished by collision: above this threshold, only the stable extinction equilibrium remained. In this model, therefore, adaptive evolution did not reduce population density gradually to zero, as in the two previous examples, but instead caused the population to go extinct abruptly. Fig. 4 illustrates this scenario.

Such abrupt transitions to extinction, caused by directional selection, have been termed 'evolutionary suicide' by Ferrière (2000). More precisely, evolutionary suicide is defined as a trait substitution sequence driven by mutation and selection that takes a population toward and across a
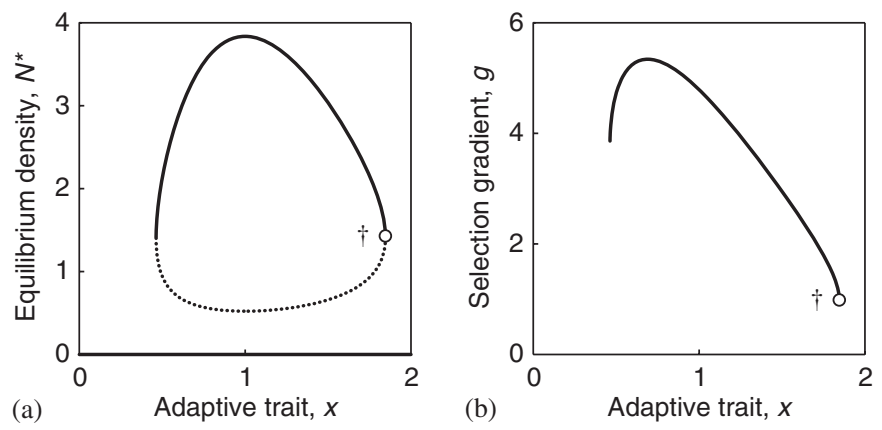

Fig. 4. Illustration of evolutionary suicide. (a) Equilibrium density $N^{*}(x)$ resulting for trait values $x$. Continuous and dotted curves depict stable and unstable equilibria, respectively. (b) Selection gradient $g(x)$ resulting for trait values $x$. For all viable initial trait values, directional selection increases $x$ up to a critical trait value at which the evolving population goes extinct. The equilibrium density, trait value, and selection gradient at which extinction occurs are indicated by open circles. Parameters: $b(x)=b_{0} x e^{-x}, \alpha(\Delta x)=1 /\left(1+e^{-\mathrm{k} \Delta x}\right), b_{0}=10, k=5$, and $d=1$.

boundary in a population's trait space beyond which the population cannot persist. Once the population's traits have evolved close enough to this boundary, variants can invade that are viable as long as the current resident trait value abounds, but that are not viable on their own. When these variants start to invade the resident population, they initially grow in density; once they have become sufficiently abundant, concomitantly reducing the former resident's density, the variants bring about their own extinction.

It thus appears that the ecological requirements for selection-driven extinction are easily met. Whenever competitive ability trades off strongly with longevity or fecundity, and competition is sufficiently asymmetric, directional selection on traits underlying competitive ability is expected to reduce population density. If the resultant densities fall below the threshold density of an allee effect, or if they imply a much elevated risk of accidental extinction, the population is doomed. The potential ubiquity of selection-driven extinctions is underscored by other examples of extinctions caused by adaptation in different traits, including anti-predator behavior (Matsuda and Abrams, 1994b), sexual traits (Kirkpatrick, 1996; Kokko and Brooks, 2003), dispersal rates (Gyllenberg et al., 2002), mutualism rates (Ferrière et al., 2002), cannibalistic traits (Dercole and Rinaldi, 2002), maturation reaction norms (Ernande et al., 2002), levels of altruism (Le Galliard et al., 2003), and selfing rates (Cheptou, 2004); see also the review by Parvinen (2006). Furthermore, Dieckmann and Ferrière (2004) showed, by examining ecologically explicit multilocus models featuring either diallelic loci or continua of alleles, that the incidence of evolutionary suicide is by no means restricted to phenotypic models of asexual evolution, but robustly occurs also when sexual inheritance is taken into account.

It is not accidental that two of the examples described in some detail above involved discontinuous transitions in population density at critical trait values. In the context of a model of dispersal evolution in metapopulations, 
Gyllenberg et al. (2002) proved that discontinuous transitions to extinction, implying catastrophic bifurcations, are a prerequisite for evolutionary suicide. This finding applies more generally: wherever a population goes to extinction through a continuous transition, it cannot undergo evolutionary suicide (Gyllenberg and Parvinen, 2001). This is easily shown for cases in which a population's density $N$ and adaptive trait $x$ are both one-dimensional (Dieckmann and Ferrière, 2004). The generic continuous transition to extinction then is the transcritical bifurcation, in which a positive equilibrium and the extinction equilibrium collide and exchange their stability at a critical trait value $x_{\mathrm{c}}$. In the vicinity of $x_{\mathrm{c}}$, population dynamics can always be written as $\left.\frac{d}{d t} N=\overline{[}\left(x-x_{\mathrm{c}}\right)-N / K\right] r N$, where $K>0$ scales $N$ and $r>0$ scales $\frac{d}{d t} N$ (up to redirection of $x$; Guckenheimer and Holmes, 1997, p. 145). With the per capita growth rate of a variant with trait value $x_{\mathrm{v}}$ in an environment with population density $N$ thus being given by $\left[\left(x_{\mathrm{v}}-x_{\mathrm{c}}\right)-\right.$ $N / K] r$, and with the equilibrium population density of a resident population with trait value $x=x_{\mathrm{c}}$ vanishing, $N=0$, we obtain the invasion fitness $f\left(x_{\mathrm{v}}, x_{\mathrm{c}}\right)=\left(x_{\mathrm{v}}-\right.$ $\left.x_{\mathrm{c}}\right) r$ for the rare variant competing with the critical resident. In addition, the consistency condition $f(x, x)=$ 0 for ecological equilibrium has to be fulfilled for all $x$. When making the generic assumption that $f\left(x_{\mathrm{v}}, x\right)$ has a leading linear order around $x_{\mathrm{v}}, x=x_{\mathrm{c}}$, i.e., $f\left(x_{\mathrm{v}}, x\right)=$ $c_{\mathrm{v}} x_{\mathrm{v}}+c x$, the coefficients $c_{\mathrm{v}}$ and $c$ can be determined from the two constraints $f\left(x_{\mathrm{v}}, x_{\mathrm{c}}\right)=\left(x_{\mathrm{v}}-x_{\mathrm{c}}\right) r$ for all $x_{\mathrm{v}}$, and $f(x, x)=0$ for all $x$, which yields $f\left(x_{\mathrm{v}}, x\right)=\left(x_{\mathrm{v}}-x\right) r$. The selection gradient operating on the adaptive trait $x$ is thus given by $\left.\frac{\partial}{\partial x_{\mathrm{v}}} f\left(x_{\mathrm{v}}, x\right)\right|_{x_{\mathrm{v}}=x}=r$, which is always positive. This means that adaptive evolution takes $x$ away from $x_{\mathrm{c}}$ by making it larger, thus increasing the equilibrium population density from $N^{*}\left(x_{\mathrm{c}}\right)=0$ to $N^{*}(x)=\left(x-x_{\mathrm{c}}\right) K$. Therefore, adaptive evolution in this system can never cause evolutionary suicide by driving $x$ toward the critical trait value $x_{\mathrm{c}}$. Similar conclusions were reached by Gyllenberg and Parvinen (2001) and by Webb (2003).

In this section we reviewed how the proper population dynamical embedding of models of adaptive life-history evolution, including both frequency-dependent and density-dependent selection pressures, results in predictions of selection-driven extinction under a wide range of ecologically plausible scenarios. We propose that the commonly accepted null hypothesis of population extinctions in the fossil record to have resulted from ecological or externally imposed environmental changes needs to be reconsidered: at the present state of knowledge, adaptive evolution cannot be ruled out as a potentially widespread agent of population extinctions.

\section{Evolutionary epidemiology cannot rely on $\boldsymbol{R}_{0}$ maximization}

In this section, we provide a concrete illustration of the very general, and hence unavoidably abstract, concept of the environmental feedback loop, by analyzing a few simple but exemplary cases. By focusing on the evolution of virulence, these examples also demonstrate the potential for mutual illumination between applied and abstract ESS theory.

For a long time, it was close to dogma in epidemiological theorizing (e.g., Anderson and May, 1982, 1991) that the main basis for the study of virulence evolution should be sought in the maximization of $R_{0}$, defined as the number of secondary infections engendered by a primary infection in an otherwise infection-free population. To this end, $R_{0}$ is considered as a function of the disease's demographic parameters, which in turn are envisaged as functions of some underlying trait vector $x$ that is supposed to be under evolutionary control.

At the opposite extreme of the abstraction spectrum, Metz et al. (1996b), extending results by Mylius and Diekmann (1995), proved that for ESSs to be characterizable in terms of an optimization principle it is necessary and sufficient that

(A) there exists a function $\varsigma: \mathbf{R} \times \mathbf{E} \rightarrow \mathbf{R}$, with $\mathbf{R}$ denoting the real numbers and $\mathbf{E}$ the realizable environmental conditions, increasing in its first argument, and a function $\psi: \mathbf{X} \rightarrow \mathbf{R}$, with $\mathbf{X}$ denoting the set of potential values of the trait vector, such that

$\operatorname{sign} \rho\left(x_{\mathrm{v}}, E\right)=\operatorname{sign} \varsigma\left(\psi\left(x_{\mathrm{v}}\right), E\right)$,

with $\rho\left(x_{\mathrm{v}}, E\right)$ denoting invasion fitness, defined as the asymptotic per capita rate of population increase of a variant with trait $x_{\mathrm{v}}$ in a resident environment $E$.

Metz et al. (1996b) also proved (A) to be equivalent to

(B) there exists a function $\eta: \mathbf{X} \times \mathbf{R} \rightarrow \mathbf{R}$, decreasing in its second argument, and a function $\phi: \mathbf{E} \rightarrow \mathbf{R}$ such that $\operatorname{sign} \rho\left(x_{\mathrm{v}}, E\right)=\operatorname{sign} \eta\left(x_{\mathrm{v}}, \phi(E)\right)$.

Conditions (A) and (B) can be paraphrased as follows: (A) means that the trait values affect fitness effectively in a one-dimensional monotone manner, and (B) means that the environment acts effectively in a one-dimensional monotone manner. The reason for the epithet 'effectively' is that the one-dimensionalness and monotonicity only need to pertain to the range of fitness values that matter in ESS considerations, i.e., to those surrounding the change from negative to positive values.

Relations (7) and (8) can be related to each other by the observation that, if an optimization principle exists,

(C) it is possible to choose the functions $\phi$ and $\psi$ such that

$\operatorname{sign} \rho\left(x_{\mathrm{v}}, E\right)=\operatorname{sign}\left(\psi\left(x_{\mathrm{v}}\right)-\phi(E)\right)$,

where $\phi$ and $\psi$ are connected through the relation

$\psi(x)=\phi\left(E_{\text {attr }}(x)\right)$

with $E_{\text {attr }}(x)$ denoting the environment engendered by any attractor attained by the community dynamics for the parameter vector $x$. This, of course, implies that a suitable function $\phi$ will yield the same value for all the attractors that may possibly by attained by $x$. With this additional notation in place, we can also be more precise about the set $\mathbf{E}$ of realizable environmental conditions: $\mathbf{E}=E_{\text {attr }}(\mathbf{X})$. For 
environments outside this set, invasion fitness $\rho$ may assume any shape, just as the supposed existence of an optimization principle does not impose any restrictions on $\rho$ except locally around the subset of $\mathbf{E} \times \mathbf{X}$ for which $\rho\left(x_{\mathrm{v}}, E\right)=0$.

To better connect with the notation used in the previous two sections, it may be helpful to observe that the environment-dependent and resident-trait-dependent notions of invasion fitness (denoted throughout this article by $\rho$ and $f$, respectively) are related to each other by $f\left(x_{\mathrm{v}}, x\right)=\rho\left(x_{\mathrm{v}}\right.$, $\left.E_{\text {attr }}(x)\right)$, for any variant trait $x_{\mathrm{v}}$ and resident trait $x$.

Naturally, results (A)-(C) hinge on the interpretation of the term 'optimization principle'. The latter is defined by Metz et al. (1996b) as a function from trait values to real numbers such that, for any possible constraint on the traits, the ESS(s) can be calculated by maximizing this function. The proviso in the previous sentence mirrors the usual practice of combining an optimization principle, derived from the population dynamics, with a discussion of the dependence of the evolutionary outcome on the possible constraints. Details of these considerations may be found in Metz et al. (1996b), available at http://www.iiasa.ac.at/ cgi-bin/pubsrch?WP96004. What matters here is that, while condition (A) is close to trivial, the equivalent condition (B) and relation (10) in condition (C) provide a useful tool for either deriving optimization principles or proving the non-existence of such principles, for large collections of population dynamical models. Below we will demonstrate their application by means of some simple examples.

Just as evolution maximizes the function $\psi$ appearing in (A), it minimizes the function $\phi$ in (B). Therefore, and since $\phi$ can be interpreted as a measure of environmental quality, the latter has been dubbed a pessimization principle by Mylius and Diekmann (1995): in the end, the worst attainable world remains, together with those types that can just cope with it.

As an aside, it may be worth pointing out that the pairwise invasibility plots for eco-evolutionary models allowing an optimization principle exhibit an immediately recognizable, very special geometry, as illustrated in Fig. 5. This geometry is a direct consequence of the linear preorder established by any optimization principle and illustrates, in a visually easily recognizable manner, the structural instability of optimization models.

The epidemiological models that we consider below have been chosen for the simplicity of the calculations they engender. In particular, their community dynamics possess unique internal point attractors (which is almost a sine qua non for obtaining analytical results). That these models also allow explicit solutions for the equilibria is a boon (when no explicit solutions are available, the same results can usually be derived through an implicit differentiation of the equilibrium equations). For a discussion of the epidemiological implications and a similar analysis of another suite of models see Dieckmann (2002).

We start out by giving a full population dynamical description of the ecological context, before reverting to considerations focusing on infected individuals. It is the individual-based dynamics of the latter that provides the basis for the classification of the environmental feedback loop based on its consequences for the ESSs of disease traits. The details of the population dynamics surrounding infected individuals is relevant only in so far as it acts as an environment affecting the population dynamical behavior of the infected individuals.

To characterize the potential instantaneous environmental conditions to which infected individuals may be exposed, we follow standard notation by letting $S$ denote the density of susceptible individuals, while $I$ denotes the density of infected individuals. After specifying the dynamics of this instantaneous environment, the corresponding evolutionary environments can be calculated from the attractors of this dynamics. Infections occur according to the simple law of mass action, with a fixed rate constant $\beta$. Infected individuals do not recover but die at a per capita rate $\alpha$, acting on top of the per capita death rate experienced by susceptible and infected individuals alike. In the absence of the disease, $I=0$, the population grows in a density-dependent manner, with per capita birth rate $b_{0}-h_{\mathrm{b}}(S, 0)$ and per capita death rate $d_{0}-h_{\mathrm{d}}(S, 0)$, with $b_{0}>d_{0}>0$. The functions $h_{\mathrm{b}}$ and $h_{\mathrm{d}}$ both increase in $S$, with $h_{\mathrm{b}}(0,0)=h_{\mathrm{d}}(0,0)=0$. The full population dynamical equations are then given by

$$
\begin{aligned}
& \frac{d S}{d t}=[b(S, I)-d(S, I)-\beta I] S, \\
& \frac{d I}{d t}=[\beta S-\alpha-d(S, I)] I,
\end{aligned}
$$

with

$b(S, I)=b_{0}-h_{\mathrm{b}}(S, I), \quad d(S, I)=d_{0}+h_{\mathrm{d}}(S, I)$.

(The implicit assumption that infected individuals are not allowed to reproduce greatly simplifies the proofs of the attractivity of the equilibria, but can probably be relaxed.) The parameters $\alpha$ and $\beta$ are assumed to be under evolutionary control by the disease (evolution in hostcontrolled traits is not considered here). As usual, we assume $\alpha$ and $\beta$ to be connected by a constraint: $\beta$ cannot become too high and $\alpha$ simultaneously not too low, which can be expressed as $g(\alpha, \beta) \leqslant m$ with $g$ increasing in $\beta$ and decreasing in $\alpha$. As evolution acts to increase $\beta$ and decrease $\alpha$, it will quickly run into this constraint. From there on, evolution will effectively be restricted to the curve $g(\alpha, \beta)=m$, alternatively parameterized as $\beta=\beta(\alpha)$, or as $(\alpha(x), \beta(x))$ for some scalar physiological trait $\mathrm{x}$.

Within the general class of models (11), we consider four exemplary cases,

(i) $h_{\mathrm{b}}(S, I)=\kappa(S+I), \quad h_{\mathrm{d}}(S, I)=0$,

(ii) $h_{\mathrm{b}}(S, I)=0, \quad h_{\mathrm{d}}(S, I)=\kappa S$,

(iii) $h_{\mathrm{b}}(S, I)=0, \quad h_{\mathrm{d}}(S, I)=\kappa S^{2}$,

(iv) $h_{\mathrm{b}}(S, I)=0, \quad h_{\mathrm{d}}(S, I)=\kappa(S+I)$. 

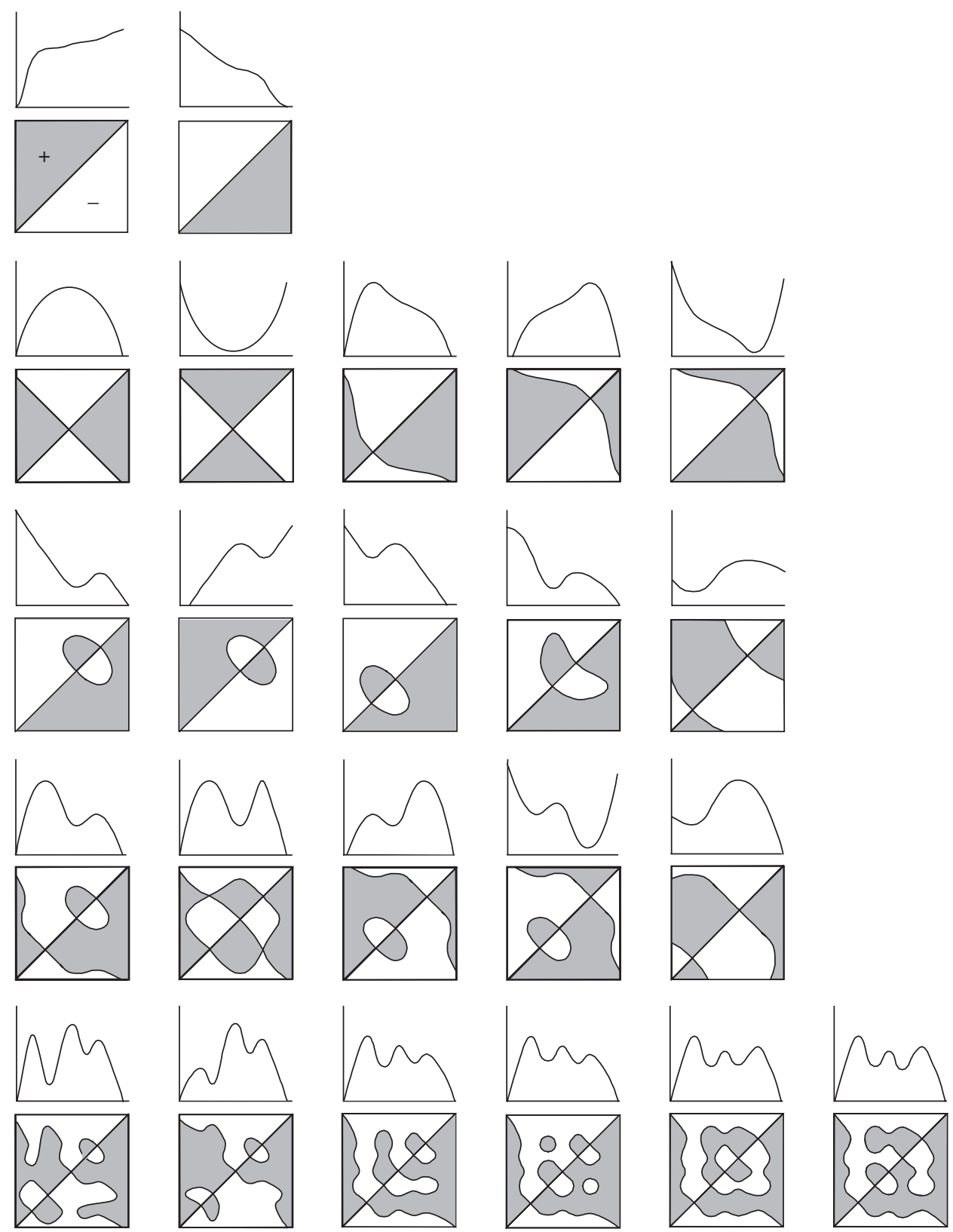

Fig. 5. Optimization principles (upper rows; horizontal axes: adaptive trait, vertical axes: quantity optimized by evolution), together with the corresponding pairwise invasibility plots (lower rows; horizontal axes: resident trait, vertical axes: variant trait). Notice that the existence of an optimization principle amounts to no more and no less than that all feasible values of the trait vector can be linearly pre-ordered: after dividing out over the equivalence relation "equally good", one obtains a linearly ordered set of equivalence classes. The geometric implications are two-fold. First, the antisymmetry of linear orders translates into the skew symmetry of the pairwise invasibility plots. Second, the transitivity of linear orders translates into the fact that any isolas of the non-diagonal zero-contour curve (these isolas correspond to local maxima of the optimization principle that are exceeded by its global maximum) have counterparts in wiggles in any other non-diagonal zero-contour curves that span the same range of trait values, either horizontally or vertically.

These model families have been rigged so that for model (i) and (ii) the environmental feedback for the disease is onedimensional monotone. According to conditions (A) and (B), these models thus support an optimization principle. For model (i) the optimization principle is equivalent (i.e., monotonically related) to $R_{0}$, while for model (ii) this is not the case. For model (iii) the environment feedback acts one-dimensionally but not monotone, and for model (iv) it acts two-dimensionally. It should be understood that the specific examples in Eq. (11c) are chosen primarily for didactical purposes. For their individual-based underpinning one may think of population regulation through fighting. For models (i) and (iv) fighting may be initiated by all individuals, whereas for models (ii) and (iii) infected individuals are assumed to suffer from fights without being able to initiate such fights themselves. Model (iii) is based 
on the assumption of aggression increasing linearly with aggressor density. Fighting, of course, may here be replaced by any other form of interference competition.

Since we only have to deal with point attractors of the community dynamics, we can use $R$, the lifetime per capita production of new disease cases by a variant disease case, as a proxy for invasion fitness. We start by expressing $R$ as a general function of the variant traits $x_{\mathrm{v}}=\left(\alpha_{\mathrm{v}}, \beta_{\mathrm{v}}\right)$ and of the variables $(S, I)$ parameterizing the potential environmental conditions,

$$
\begin{aligned}
& \text { (i) } R\left(\alpha_{\mathrm{v}}, \beta_{\mathrm{v}} ; S, I\right)=\frac{\beta_{\mathrm{v}} S}{\alpha_{\mathrm{v}}+d_{0}}, \\
& \text { (ii) } R\left(\alpha_{\mathrm{v}}, \beta_{\mathrm{v}} ; S, I\right)=\frac{\beta_{\mathrm{v}} S}{\alpha_{\mathrm{v}}+d_{0}+\kappa S}, \\
& \text { (iii) } R\left(\alpha_{\mathrm{v}}, \beta_{\mathrm{v}} ; S, I\right)=\frac{\beta_{\mathrm{v}} S}{\alpha_{\mathrm{v}}+d_{0}+\kappa S^{2}}, \\
& \text { (iv) } R\left(\alpha_{\mathrm{v}}, \beta_{\mathrm{v}} ; S, I\right)=\frac{\beta_{\mathrm{v}} S}{\alpha_{\mathrm{v}}+d_{0}+\kappa(S+I)} .
\end{aligned}
$$

It is only later that we will confine attention to the realizable environments, given by the equilibrium values $\left(S^{*}(\alpha, \beta), I^{*}(\alpha, \beta)\right)$ produced by the possible residents $x=(\alpha, \beta)$.

To derive firm conclusions from (12), we have to make sure that the dynamical equations (11) indeed have unique equilibrium points as their only internal attractors. This appears indeed to be the case for models (i), (ii), and (iv). For model (iii), bistability can occur, with the state space divided into the basin of an internal locally stable equilibrium and the basin of the disease-free boundary equilibrium. Since the statements about ESSs to be made below are predicated on the presence of the disease, these stay true, though vacuous, in the absence of that disease. All conclusions to be derived from (12) will thus be valid.

For model (i), $R$ increases with $S$. So the optimization principle can be constructed directly from (10). Minimizing $S^{*}$, which can easily be seen from (11) to yield $S^{*}=$ $\left(\alpha+d_{0}\right) / \beta$, should thus be equivalent to maximizing $\psi(\alpha, \beta)=-S^{*}=-\left(\alpha+d_{0}\right) / \beta$. To calculate $R_{0}$ for this model, we observe that $R_{0}(\alpha, \beta)=R\left(\alpha, \beta ; S_{0}, 0\right)=\beta S_{0} /$ $\left(\alpha+d_{0}\right)$, with $S_{0}$ denoting the equilibrium value for $S$ in the absence of the disease. It is not difficult to see that $R_{0}$ and the $\psi$ resulting from our general construction are indeed monotonically related, independently of the value of $S_{0}$.

For model (ii), $R$ is again monotone in $S$. With $S^{*}=\left(\alpha+d_{0}\right) /(\beta-\kappa)$, we find that two equivalent optimization principles can be constructed as counterparts in trait space of minimizing $S^{*}: \psi=-\left(\alpha+d_{0}\right) /(\beta-\kappa)$ and $\tilde{\psi}=(\beta-\kappa) /\left(\alpha+d_{0}\right)$. However, neither of these is equivalent to maximizing $R_{0}=\beta S_{0} /\left(\alpha+d_{0}+\kappa S_{0}\right)=\beta\left(b_{0}-\right.$ $\left.d_{0}\right) /\left[\kappa\left(\alpha+b_{0}\right)\right]$, where we used $S_{0}=\left(b_{0}-d_{0}\right) / \kappa$ as for model (i). To see this non-equivalence, it suffices to observe that the contour lines, defined by $R_{0}(\alpha, \beta)=R_{0}\left(\alpha_{0}, \beta_{0}\right)$ and $\psi(\alpha, \beta)=\psi\left(\alpha_{0}, \beta_{0}\right)$ for given $\left(\alpha_{0}, \beta_{0}\right)$, differ, as can be seen from the lack of coincidence in their derivatives at $\left(\alpha_{0}, \beta_{0}\right)$, calculated via an implicit differentiation of the defining relations: $\mathrm{d} \alpha / \mathrm{d} \beta=\left(\kappa \alpha_{0}+b_{0}\right) /\left(\beta_{0} \kappa\right)$ for $R_{0}$, which differs from $\mathrm{d} \alpha / \mathrm{d} \beta=\left(\alpha_{0}+d_{0}\right) /\left(\beta_{0}-\kappa\right)$ for $\psi$.

The fact that invasion fitness in model (iii) is nonmonotone in any possible single scalar summary of the condition of the environment, and that the evolutionary environment in model (iv) is essentially two-dimensional, can already be guessed from (12). However, to prove these statements, we have to deal with the fact that, for instance, in model (iii) $R$ should be non-monotone relative to whatever summary variable, if its domain is restricted to the realizable values of $S$ and in addition to an infinitesimal neighborhood of those combinations of $\left(\alpha_{\mathrm{v}}, \beta_{\mathrm{v}}\right)$ and $S^{*}(\alpha, \beta)$ for which $R\left(\alpha_{\mathrm{v}}, \beta_{\mathrm{v}} ; S^{*}(\alpha, \beta)\right)=1$. Doing so involves many technicalities. These are collected in Appendix A. The reason for going through the motions there is that the utilized techniques are representative for a class of techniques that allow dealing with much more difficult problems of a similar ilk.

For the more biologically oriented reader, we add a small dessert in the form of a fifth model. The fact that for models (ii)-(iv) the ESS cannot be calculated by maximizing $R_{0}$ may not pass the naive practitioner unnoticed, as in these models maximization of $R_{0}$ gives the counterintuitive result that the outcome of the maximization depends in an essential manner on the value of $S_{0}$. So the $R_{0}$-maximization strategy seems harmless: just maximize $R_{0}$, and if you cannot do so independently of $S_{0}$, start thinking a little better. Our last example is specifically geared to deal with this potential objection against our denouncement of $R_{0^{-}}$ maximization (for further corroborative examples, see Dieckmann, 2002). The fifth model is defined by

$$
\text { (v) } \begin{aligned}
\frac{d S}{d t} & =\left[b(S, I)-d_{0}-\beta I\right] S, \\
\frac{d I}{d t} & =\left[\beta S-\alpha(1+\theta I)-d_{0}\right] I,
\end{aligned}
$$

with

$b(S, I)=b_{0}[1-k(S+I)]$,

i.e., here the disease-dependent mortality increases with the severity of the infection in the population. As a tongue-incheek explanation, one may think of a reduction in the efficiency of the health-care system occurring when too many people are infected. This model again has a unique internal point attractor. Our proxy of invasion fitness is given by

(v) $R\left(\alpha_{\mathrm{v}}, \beta_{\mathrm{v}} ; S, I\right)=\frac{\beta_{\mathrm{v}} S}{\alpha_{\mathrm{v}}(1+\theta I)+d_{0}}$.

In an as yet uninfected population, this reduces to the usual $R_{0}(\alpha, \beta)=\beta S_{0} /\left(\alpha+d_{0}\right)$, as in model (i). So here maximizing $R_{0}$ gives a result that is independent of $S_{0}$, which means that the non-applicability of $R_{0}$-maximization may easily elude the naive practitioner. However, since the feedback environment in model (v) is two-dimensional, there exists no optimization principle, and the ESS cannot be 
calculated by maximizing $R_{0}$. All that remains in cases like this is to do a full ESS calculation based on the fitness proxy (14) and the equilibrium solutions

$$
\text { (v) } \begin{aligned}
S^{*}(\alpha, \beta) & =\frac{(\beta+k)\left(\alpha+d_{0}\right)-\theta \alpha\left(b_{0}-d_{0}\right)}{\beta(\beta+k)+\theta \alpha k}, \\
I^{*}(\alpha, \beta) & =\frac{\beta S^{*}(\alpha, \beta)-\alpha-d_{0}}{\theta \alpha} .
\end{aligned}
$$

Also for models (iii) and (iv), such a full ESS calculation could be carried out based on using the equilibrium solutions of these models,

(iii) $S^{*}=\frac{\beta-\sqrt{\beta^{2}-4 \kappa\left(\alpha+d_{0}\right)}}{2 \kappa}$,

(iv) $I^{*}=\frac{\left(b_{0}-d_{0}\right)(\beta-\kappa)-\alpha-d_{0}}{\beta^{2}-\kappa^{2}+\kappa}$,

$$
S^{*}=\frac{\alpha-d_{0}+\kappa I^{*}}{\beta-\kappa} .
$$

Eqs. (15) bring out the unfortunate consequence of having to rely on a full ESS calculation: for even slightly more complicated models, the ensuing formulas have a tendency to be rather opaque, to say the least.

The overall take-home message of this section perhaps does not come as a surprise in a special issue on ESS theory: simple optimization can rarely be used to predict evolutionary outcomes - and even when an optimization principle exists, it is rarely equivalent to $R_{0}$ (see also Mylius and Diekmann, 1995). This general conclusion applies to evolutionary epidemiology in particular (Dieckmann, 2002), where $R_{0}$ maximization ruled maybe even more firmly than in other areas of population biology. Conditions (A) and (B) provide a complete characterization, phrased in terms of the properties of the environmental feedback loop, of all cases in which an optimization principle does a proper job. In addition, relation (10) in condition (C) provides a useful tool for getting hold of such an optimization principle if one exists.

\section{Generically, Hardy-Weinberg ratios occur for evolutionary reasons only}

The fourth of the surprises brought about by the incorporation of more realistic environmental feedback loops into evolutionary models is wholly conceptual, without an immediate unexpected biological phenomenon in its wake. The reason is that the evolutionary phenomenon to be discussed in this section has been unwittingly presaged by a standard textbook simplification, which, however, rarely applies in ecological reality. In almost any textbook chapter on the mathematics of selection for randomly mating populations, the Hardy-Weinberg law at the level of new zygotes is presented as a useful generalization, introduced and motivated from a purely mechanistic basis. In contrast to this treatment, we will show below that, even when assuming the global random union of gametes, almost no population dynamical model with ecologically realistic life histories has its zygotic genotype frequencies on the Hardy-Weinberg manifold. Yet, even in those cases, Hardy-Weinberg frequencies may well be seen in practice. The apparent contradiction between the preceding two statements is resolved by the demonstration, to be given below, that the exceptional parameter values necessary for Hardy-Weinberg frequencies occur as ESSs in a large class of ecologically more realistic models. Thus, the Hardy-Weinberg law may indeed reign in nature, but for evolutionary instead of purely mechanistic reasons.

In textbooks on evolutionary biology, it is close to dogma that under the random union of gametes, be it due to a mixing of gametes in broadcast spawners or to random mating, the newly formed zygotes occur in Hardy-Weinberg proportions. However, as is neatly stressed for the case without selection in the unpublished textbook by Felsenstein (1978-2005), available at http://evolution. genetics.washington.edu/pgbook/pgbook.html, the HardyWeinberg law for zygotes only holds when allele frequencies in the two sexes are equal (without selection, the zygotic genotype frequencies relax to Hardy-Weinberg proportions in one generation, at least in the case of autosomal genes). With selection, it should not be so much the allele frequencies in the two sexes that should be equal, but the allele frequencies in their gametic outputs. This is where ecological considerations kick in.

The condition for generically having equal micro- and macrogametic allele frequencies is that, for all feasible environmental trajectories, the expected micro- and macrogametic outputs in the different genotypes are proportional at all ages. If we restrict attention to equilibria, a proportionality of the lifetime outputs is sufficient. Although customarily assumed, such proportionality is actually exceptional, when seen against the background of most life histories encountered in the field. We may think, for instance, of a life history in which females reproduce for the first time at age 1, and males at age 2, and otherwise produce age-independent gametic output. When considering an age-independent annual density-dependent survival $s$, we can envisage a mutant allele that, in the heterozygote, changes this survival by a factor $\alpha$. Then, at any prescribed density, carrying one copy of this mutation changes the lifetime gametic output of females by a factor $\alpha \frac{1-s}{1-\alpha . s}$, and that of males by a factor $\alpha^{2} \frac{1-s}{1-\alpha s^{s}}$. To achieve proportionality of the macro- and microgametic outputs of the different genotypes, the ratio of these two factors must be 1. As long as both alleles are present, the genotype frequencies in the newly produced zygotes do not lie on the Hardy-Weinberg manifold. In particular, if the invasion ends in a stable polymorphism, this departure from Hardy-Weinberg frequencies persists. Similar statements apply essentially whenever the age dependence of micro- and macrogametic production is not exactly in proportion. This is even so in hermaphroditic annual plants-which may be perceived as the example best conforming to the simplified ecology of the population genetics textbooks - when genetic 
differences affect seed production relative to flower production. The latter would apply, in particular, to any adaptive trait affecting relative competitive ability during the seed-setting phase.

Observationally, transient polymorphisms are probably much less important than polymorphic ESSs. In recent days, it has been become clear, i.a. from evolutionary game theory (Maynard Smith, 1982), from the consideration of fluctuating environments (e.g., Ellner, 1996), and most recently as a result of the adaptive dynamics research program (Metz et al., 1996a; Geritz et al., 1998; Doebeli and Dieckmann, 2000), that realistic ecologies more often than not are conducive to the generation of diversity. In some cases, such diversity is realized through species formation (Dieckmann et al., 2004), but on many other occasions some within-species form of diversity results, be it purely phenotypic or genetically based (Leimar, 2005). It is this genetically based diversity that has our interest here.

We will illustrate our point with a very simple ecogenetic model. To that end, we concentrate on an annual organism with a potentially polymorphic locus with two segregating alleles, leading to phenotype vectors $x_{G}$, with $G=a a, a A, A A$. The expected macrogametic output of an individual with phenotype $x$ is given by $\lambda(x, E)$, where $E$ denotes the instantaneous ecological environment. Similarly, the expected microgametic output is given by $\mu(x, E)$. In this way, we may incorporate any determination of sexual roles, from hermaphroditism to genetically determined dioicy. The environment $E$ may, for instance, be an $m$-dimensional vector,

$E=F\left(\sum_{G} \phi_{1}\left(E, x_{G}\right) n_{G}, \ldots, \sum_{G} \phi_{k}\left(E, x_{G}\right) n_{G}\right)$,

where $n_{G}$ denotes the population density of genotype $G$. For the $\phi_{i}$ one may think of the per capita use of resources like light, water, space, and various nutrients. The function $F$ then represents the outcome of the resource dynamics given these demands. When the frequency of $A$ in the micro- and macrogametic outputs is denoted by $p_{A}$ and $q_{A}$, respectively, then, under the assumption of random mating, we have

$n_{a a}=p_{a} q_{a} N, \quad n_{a A}=\left(p_{A} q_{a}+p_{a} q_{A}\right) N$,

$n_{A A}=p_{A} q_{A} N$,

where $N=n_{a a}+n_{a A}+n_{A A}$ denotes total population density, and $p_{a}=1-p_{A}$ and $q_{a}=1-q_{A}$. Moreover, with next generation values denoted by $p_{A}^{\prime}$ and $q_{A}^{\prime}, p_{A}$ and $q_{A}$ satisfy the recurrences

$\bar{\mu} p_{A}^{\prime}=\mu_{A A} p_{A} q_{A}+\frac{1}{2} \mu_{a A}\left(p_{A} q_{a}+p_{a} q_{A}\right)$,

$\bar{\lambda} q_{A}^{\prime}=\lambda_{A A} p_{A} q_{A}+\frac{1}{2} \lambda_{a A}\left(p_{A} q_{a}+p_{a} q_{A}\right)$,

where $\mu_{\mathrm{G}}$ and $\lambda_{\mathrm{G}}$ are abbreviations for $\mu\left(x_{G}, E\right)$ and $\lambda\left(x_{G}, E\right)$, respectively, and

$\bar{\mu}=p_{A} q_{A} \mu_{A A}+\left(p_{A} q_{a}+p_{a} q_{A}\right) \mu_{a A}+p_{a} q_{a} \mu_{a a}$,
$\bar{\lambda}=p_{A} q_{A} \lambda_{A A}+\left(p_{A} q_{a}+p_{a} q_{A}\right) \lambda_{a A}+p_{a} q_{a} \lambda_{a a}$.

As a matter of convenience, we absorb all density regulation in $\lambda$, i.e., we include in $\mu$ fertilizing propensities, but not realized effectivities in the form of offspring numbers, so that we can do the full zygote-to-zygote bookkeeping through $\lambda$. For the total population density we thus obtain the recurrence

$N^{\prime}=\bar{\lambda} N$,

which completes the specification of our eco-genetic model.

Based on the setting captured by (16)-(18), we can now examine under what conditions the new zygote genotype frequencies will stay on the Hardy-Weinberg manifold. As can be seen from (16b), this requires $p_{A}^{\prime}=q_{A}^{\prime}$ in (17), for all relevant allele frequencies and population densities. The latter is ensured if $\lambda_{G}=\theta \mu_{G}$ for all $G$, but generally does not apply otherwise.

More important than the recurrences themselves are the equilibria they engender. These can be calculated from (16) to (18) after dropping the primes. When we refer to (16)-(18) below, it will be assumed, unless mentioned otherwise, that the primes have been dropped and that, accordingly, $p_{A}$ and $q_{A}$ denote equilibrium values.

The seeming oversimplification of the model specified by (16)-(18) is justified by the fact that it is actually much less special than our initial description suggests. Following arguments initiated by Charlesworth $(1976,1994)$, it was shown by Diekmann et al. (2003) that, under the assumption of random mating, the same equilibrium equations follow from a large class of physiologically structured population models. For this, we have to interpret $\lambda$ as the expected lifetime macrogametic output from a new zygote times the probability of their fertilization, $\mu$ as the expected lifetime microgametic output times their fertilization propensity, and $n_{G}$ and $N$ as birth rates. The simple and the general case of course differ in their internal, i.e., population dynamical, stability properties. However, when it comes to external stability, i.e., the stability towards invasion by variants altering phenotypic expression, the two cases coincide, since all that matters in both cases is whether a generation-based linearized recurrence for the frequency of variant heterozygotes predicts their increase or decrease.

The system of equilibrium equations (16)-(18) allows, in principle, two different classes of equilibria, characterized by the routes one can follow in the solution process. Along the first route, one assumes that at least two of the $\lambda_{\mathrm{G}}$ or two of the $\mu_{\mathrm{G}}$ differ. In that case, given the $\lambda_{\mathrm{G}}$ and $\mu_{\mathrm{G}}$, (17) produces up to three isolated internal solutions for $p_{A}$ and $q_{A}$ (these solutions have been extensively studied by Owen, 1952; Bodmer, 1965; and Mandel, 1971; see also Karlin and Lessard, 1986 and Diekmann et al., 2003). We will call these equilibria 'population genetic' solutions. The second route is based on the possibility, first discussed by Lewontin (1958), of a solution in which all $\lambda_{\mathrm{G}}$ are equal, and so are all $\mu_{\mathrm{G}}$. We will call these equilibria 
'feedback-driven' solutions. Any feedback-driven solution satisfies the alternative equilibrium equations

$\lambda_{a a}=\lambda_{a A}=\lambda_{A A}=1$,

$\mu_{a a}=\mu_{a A}=\mu_{A A}$,

together with (16). Whereas for a population genetic equilibrium the number of equations, $m+2+1$ for Eqs. (16)-(18) together, neatly matches the number of unknowns, $E, p_{A}$ and $q_{A}$, and $N$, this is not the case for feedback-driven equilibria, since (19) actually contains five equalities that need to be satisfied. This means that, generically, there is no solution, except for special cases, such as when it is assumed that $x_{a A}=x_{A A}$, so that both $\lambda_{a A}=\lambda_{A A}$ and $\mu_{a A}=\mu_{A A}$ hold a priori. Thus, if there are any polymorphic equilibria, we may expect them to be population genetic ones. Consequently, except in the equally non-generic case that $\lambda_{G}=\theta \mu_{G}$ for $G=a a, a A$, $A A$, the zygotic genotype frequencies are off the HardyWeinberg manifold.

The previous considerations were based on the assumption that the phenotypes $x_{G}$ are given a priori. However, in nature traits do not just take on any values, but are shaped by evolution. There are two ways in which the $x_{G}$ may change evolutionarily. Either some new allele $\alpha$ appears on the scene, or an expression modifier, denoted by $B$, appears at a locus that previously only carried an allele $b$. For the sake of concreteness, and since the loci underlying the expression of phenotypes $x$ are likely to extend beyond the single locus considered so far, we shall proceed on the assumption that the evolution of $x_{G}$ is primarily driven by modifiers. Accordingly, we mentally promote our focal locus to the status of a genetic switch, with three states, $a a$, $a A$, and $A A$, and assume that the output of this switch to a phenotypic expression is under evolutionary control. One particular reason for this ploy is that it will allow us to discuss more easily the so-called 'ideal free' ESSs (Bulmer, 1994; see also Fretwell and Lucas, 1970).

To examine the evolution of phenotypic expression, we need to consider the invasion fitness of the modifiers. For this we shall use a fitness proxy, denoted $R_{B}$, with this notation intended to stress the proxy's interpretation as an expected lifetime offspring number sensu Diekmann et al. (1990). In principle, the $B$ allele can be transmitted in four different states, in a macro- or a microgamete, together with either $a$ or $A$, and the appropriate (but necessarily complicated) procedure would be to go through a bookkeeping argument to derive the linearized recurrences for the four corresponding frequencies, followed by a calculation of $R_{B}$ as the associated dominant eigenvalue. Luckily, we can fall back on a shortcut invented by Eshel and Feldman (1984), and worked out for the most general case by Liberman (1988), who showed that $R_{B}$ can be written as a weighted sum,

$$
\begin{aligned}
R_{B}= & \frac{1}{2}\left(w_{1} \tilde{\mu}_{B b a a}+w_{2} \tilde{\mu}_{B b a A}+w_{3} \tilde{\mu}_{B b A A}\right. \\
& \left.+w_{1} \tilde{\lambda}_{B b a a}+w_{2} \tilde{\lambda}_{B b a A}+w_{3} \tilde{\lambda}_{B b A A}\right)
\end{aligned}
$$

with weights

$$
\begin{aligned}
& w_{1}=p_{B, a} q_{a}+p_{a} q_{B, a}, \\
& w_{2}=p_{B, a} q_{A}+p_{B, A} q_{a}+p_{a} q_{B, A}+p_{A} q_{B, a}, \\
& w_{3}=p_{B, A} q_{A}+p_{A} q_{B, A},
\end{aligned}
$$

where $\tilde{\lambda}=\lambda / \bar{\lambda}$ and $\tilde{\mu}=\mu / \bar{\mu}$. The averages $\bar{\lambda}, \bar{\mu}$, as well as the gamete frequencies $p_{a}, q_{a}, p_{A}, q_{A}$, are to be determined from (16) to (18) for the resident equilibrium. The longterm relative frequencies of the different transmission states of $B, p_{B, a}, q_{B, a}, p_{B, A}$, and $q_{B, A}$, are to be calculated as the components of the normalized eigenvector of the linear recurrences mentioned above. For the considerations below, there is no need to determine the weights $w_{i}$; it suffices to know that all of them are positive, that $\Sigma_{i} w_{i}=1$, and that, necessarily, $R_{b}=1$.

Armed with the fitness proxy given by (20), we can now introduce the idea of 'ideal free' ESSs. If there are no genetic constraints, in the sense that for any feasible phenotype there exists a potential modifier realizing it, the fitness contributions through the different routes-here corresponding to the states of the genetic switch - should be equal, i.e., $\tilde{\mu}_{b b a a}+\tilde{\lambda}_{b b a a}=\tilde{\mu}_{b b a A}+\tilde{\lambda}_{b b a A}=\tilde{\mu}_{b b A A}+\tilde{\lambda}_{b b A A}$. For, if they were not, then a modifier $B$ that were to change the expression of all phenotypes with a smaller fitness contribution to that of the phenotype with the highest contribution would have an $R_{B}>1$. (Let us assume, for the sake of the argument, that $\tilde{\mu}_{b b a a}+\tilde{\lambda}_{b b a a}<\tilde{\mu}_{b b a A}+\tilde{\lambda}_{b b a A}$ and $\tilde{\mu}_{b b a A}+\tilde{\lambda}_{b b a A}>\tilde{\mu}_{b b A A}+\tilde{\lambda}_{b b A A}$; then changing both $x_{b b a a}$ and $x_{b b A A}$ to $x_{b b A A}$ would lead to $R_{B}=\frac{1}{2} \sum_{i} w_{i}\left(\tilde{\mu}_{b b a A}+\tilde{\lambda}_{b b a A}\right)=$ $\frac{1}{2}\left(\tilde{\mu}_{b b a A}+\tilde{\lambda}_{b b a A}\right)>R_{b}=1$, independently of the values of the weights $w_{i}$ for either $b$ or $B$.) The notion of 'ideal free' ESSs is customarily used to set apart ESSs that equalise fitness contributions obtained through different routes, reflecting the fact that such ESSs often obtain in the ideal situation of a total freedom from constraints. (The only constraints that matter here are genetic ones; constraints on trait values remain allowed when considering ideal free ESSs.)

The just introduced ideal free ESSs do not yet satisfy the proportionality conditions $\mu_{b b a a}=\theta \lambda_{b b a a}, \mu_{b b a A}=\theta \lambda_{b b a A}$, $\mu_{b b A A}=\theta \lambda_{b b A A}$, and $\mu_{b b A A}=\theta \lambda_{b b A A}$. To ensure this, we need the additional assumption (to be called IF in the remainder of this section) that gene expression is allowed to be sex-dependent and that we can write $x_{G}=\left(x_{m, G}, x_{f, G}\right)$, $\mu\left(x_{G}, E\right)=\breve{\mu}\left(x_{m, G}, E\right), \lambda\left(x_{G}, E\right)=\breve{\lambda}\left(x_{f, G}, E\right)$, without any physiological constraints tying $x_{m, G}$ to $x_{f, G}$ and without any hard restriction on the realization of the feasible combinations of $x_{m, G}$ and $x_{f, G}$ by single mutational steps. For such "even more ideal free" ESSs, or IF-ESSs, we have $\lambda_{a a}=\lambda_{a A}=\lambda_{A A}$ and $\mu_{a a}=\mu_{a A}=\mu_{A A}$, by the same argument as given earlier. Consequently, once an IF-ESSs has been attained by evolution, zygotic gene frequencies will follow the Hardy-Weinberg law.

Another matter is that for continuous trait spaces the probability of directly jumping into an ideal free ESS or IFESS will generally be negligible, to the extent of being 
observationally irrelevant. Therefore, the question remains whether IF-ESSs are locally evolutionarily attracting. Determining attractivity is easy for one-dimensional strategy spaces (e.g., Taylor, 1989; Christiansen, 1991; Metz et al., 1996a; Eshel et al., 1997; Geritz et al., 1998), but very difficult otherwise. Leimar (2001, in press) provides a solution for cases in which, close to an ESS, mutational steps are still sufficiently small relative to the distance from that ESS that the analysis can be based on the canonical equation of adaptive dynamics (Dieckmann and Law, 1996; Durinx and Metz, 2005; Champagnat et al., this issue). In principle, convergence to the ESS may depend on the mutational covariance matrix. However, Leimar (2001, in press) derived conditions characterizing those cases in which convergence, or divergence, is determined only by the dependence of the selective regime on the ecology. These conditions are expressed in terms of local derivatives of the invasion fitness function at the ESS. (In this context, it may be worth noting that we have derived a general canonical equation for the modifierdriven evolution scenario described above; De Kovel and Metz, in preparation.) Unfortunately, the very idea of ideal free ESSs, and thus also of IF-ESSs, is based on the assumption that mutational steps are not small: in the argument leading up to the definition of ideal free ESSs, we had to assume the potential occurrence of mutational steps that change the phenotype at one setting of the genetic switch to the phenotype occurring at another setting. In Appendix B, we sketch a research program that we believe may, in the long run, resolve the convergence issue, at least in principle, based on the assumption that the mutation distribution is sufficiently smooth in phenotype space.

In this section we have argued that, once an IF-ESS has been reached evolutionarily, zygotic allele frequencies will lie on the Hardy-Weinberg manifold. Although the Hardy-Weinberg law may thus reign in nature, using it as a widely applicable primary assumption based on purely mechanistic reasons is misleading, in particular when sexstructured populations are embedded in realistic ecological settings. We have also argued that, in general, HardyWeinberg frequencies will not apply during the evolutionary transients leading to an IF-ESS. That the law applies after these transients are over, is caused by a lucky combination of environmental feedback with sufficient developmental and genetic freedom. Given such conditions, long-term evolution either keeps moving, or engenders polymorphisms that give the appearance of being selectively neutral $\left(\lambda_{a a}=\lambda_{a A}=\lambda_{A A}\right.$ and $\left.\mu_{a a}=\mu_{a A}=\mu_{A A}\right)$, so that, even for complicated life histories, the classical arguments of Hardy (1908) and Weinberg (1908) hold sway at the zygotic level.

\section{Summary}

All intelligent modeling and theorizing rely on idealizations. Good models, just as successful theories, require stripping away the non-essential, to allow an unobstructed view onto a phenomenon's core. To delineate the domain of validity of models and theories, and thus to assess whether perhaps more than the non-essential has been stripped away, robustness checks are to be carried out.

Here we have shown, in a sequence of four robustness checks, how the incorporation of enhanced degrees of ecological realism results in evolutionary phenomena not predicted by the underlying simplified models:

- When evolutionary matrix games are embedded in more realistic ecological settings, mixed ESSs no longer render neutral all involved pure strategies and their mixtures. By overcoming this fundamental structural instability, more conclusive predictions of evolutionary outcomes can be made, with population-level polymorphisms of individual-level mixed strategies becoming amenable to analysis (Section 2).

- When realistic types of density- and frequency-dependent selection are considered in models of life-history evolution, adaptations can no longer be assumed to maximize a population's viability. Instead, adaptive evolution can become responsible for bringing about a population's extinction under a variety of ecologically plausible scenarios (Section 3).

- When models of disease evolution are equipped with realistic ecological detail, attempts at predicting evolutionary outcomes through optimization principles typically become futile. As soon as the effective dimension of the feedback loop governing the interaction of an evolving population with its environment exceeds 1 , optimization-based predictions will necessarily be in error (Section 4).

- When studying the population genetics of sex-structured populations in realistic ecological settings, the Hard$\mathrm{y}$-Weinberg law for zygotic proportions loses it validity. It can be shown, however, that adherence to this law may be reestablished in the course of evolution, provided the underlying genetic system possesses sufficient flexibility (Section 5).

In all these robustness checks, the investigated perturbations of the simplified models have qualitative implications: loss of neutrality (Section 2), loss of viability (Section 3), loss of optimality (Section 4), and loss of Hardy-Weinberg proportions (Section 5). In some circumstances the tiniest perturbations suffice (Section 2), in other cases the amplitude of the new phenomena grow with the considered perturbation (Sections 4 and 5), and sometimes perturbations may need to exceed a threshold level (Section 3).

Obviously, the failed robustness checks documented in this study must not be misinterpreted as rendering the underlying simplified models useless. Instead, great care ought be taken not to abuse these idealized models by drawing biological inferences that fall outside their documented domain of validity. Since the ecological theater of most evolutionary plays occurring in nature is 
complex, that may be a tall order. Modern ESS theory is increasingly living up to this challenge.

\section{Appendix A. Ascertaining the dimension of the eco-evolutionary feedback}

To assess the monotonicity or the effective dimension of the feedback loop in the evolutionary models of epidemiological dynamics discussed in Section 4, we need to examine the dependence of $R\left(\alpha_{\mathrm{v}}, \beta_{\mathrm{v}} ; S^{*}(\alpha, \beta), I^{*}(\alpha, \beta)\right)$ on the resident trait $(\alpha, \beta)$ locally around those combinations $(\alpha, \beta)$ that yield $R\left(\alpha_{\mathrm{v}}, \beta_{\mathrm{v}} ; S^{*}(\alpha, \beta), I^{*}(\alpha, \beta)\right)=1$, for all values of the variant trait $\left(\alpha_{\mathrm{v}}, \beta_{\mathrm{v}}\right)$.

To show that in model (iii) the feedback loop is nonmonotone, we start from (12) and (15). $R$ depends on $E=$ $(S, I)$ and therefore on $(\alpha, \beta)$ in a one-dimensional manner, through $S$ alone. $R$ as a function of $S$ has a maximum at $S_{\max }\left(\alpha_{\mathrm{v}}, \beta_{\mathrm{v}}\right)=\sqrt{\left(\alpha_{\mathrm{v}}+d_{0}\right) / \kappa}$, and possesses no other internal extrema. We now consider the set $M$ of resident trait values that as residents maximize $R, \quad M=$ $\left\{(\alpha, \beta) \mid S_{\max }(\alpha, \beta)=S^{*}(\alpha, \beta)\right\}$. For $(\alpha, \beta) \in M$ we have $R\left(\alpha, \beta ; S_{\max }(\alpha, \beta)\right)=1$, and therefore $\operatorname{sign} \ln R(\alpha, \beta$; $\left.S_{\max }(\alpha, \beta)\right)=\operatorname{sign} \rho\left(\alpha, \beta ; S_{\max }(\alpha, \beta)\right)=0$. (To ward off potential confusion, we repeat that the maximization is with respect to the resident trait $(\alpha, \beta)$, and not with respect to the variant trait $\left(\alpha_{\mathrm{v}}, \beta_{\mathrm{v}}\right)$, as is common in ESS calculations.) The elements of $M$ are thus precisely those trait values around which the potentially interesting things happen: they mark the traits for which $R$ fails to be locally monotone in $S$, and the variants close to these straddle the border between positive and negative values of $\ln (R)$. The monotonicity or non-monotonicity of $R$ in $S$ is of concern only in the neighborhood of $M$. The salient point here is that $M$ does not consist of just a single point but is a onedimensional manifold, as can be seen from its definition. Any function $\phi: S \mapsto \phi(S) \in \mathbf{R}$ having a non-decreasing relation with $\operatorname{sign} \ln \left(R\left(\alpha_{\mathrm{v}}, \beta_{\mathrm{v}} ; S\right)\right.$, considered as a function of $S$ for a given $\left(\alpha_{\mathrm{v}}, \beta_{\mathrm{v}}\right) \in M$, should have its maximum at $S_{\max }\left(\alpha_{\mathrm{v}}, \beta_{\mathrm{v}}\right) \in M$-as in the close neighborhood of $M$, by the very construction of that manifold, we have $\operatorname{sign} \ln \left(R\left(\alpha_{\mathrm{v}}, \beta_{\mathrm{v}} ; S\right)=0\right.$ for $S=S_{\max }\left(\alpha_{\mathrm{v}}, \beta_{\mathrm{v}}\right)$ and $\operatorname{sign} \ln \left(R\left(\alpha_{\mathrm{v}}, \beta_{\mathrm{v}} ; S\right)=-1\right.$ for $S \neq S_{\max }\left(\alpha_{\mathrm{v}}, \beta_{\mathrm{v}}\right)$. Accordingly, there can be a single function $\phi$ fulfilling condition (B) for all $\left(\alpha_{\mathrm{v}}, \beta_{\mathrm{v}}\right) \in M$ only if $S_{\max }\left(\alpha_{\mathrm{v}}, \beta_{\mathrm{v}}\right)$ is constant on $M$. The latter, however, is not the case. Hence, model (iii) does not allow an optimization principle.

In models (i)-(iii), where the feedback loop acts through $S$ alone, it is immediately clear that the dimension of the feedback is 1 . In model (iv), where $R$ is also influenced by $I$, it is necessary to be more precise. For when the evolving population itself appears in the feedback, the very fact that there may be more than one type present in that population may increase the number of environmental variables that it is necessary to keep track of. However, the fact that infected individuals were treated on a par with susceptible individuals, through the sum $S+I$, in their influence on the density-dependent death rate, strongly suggests treating

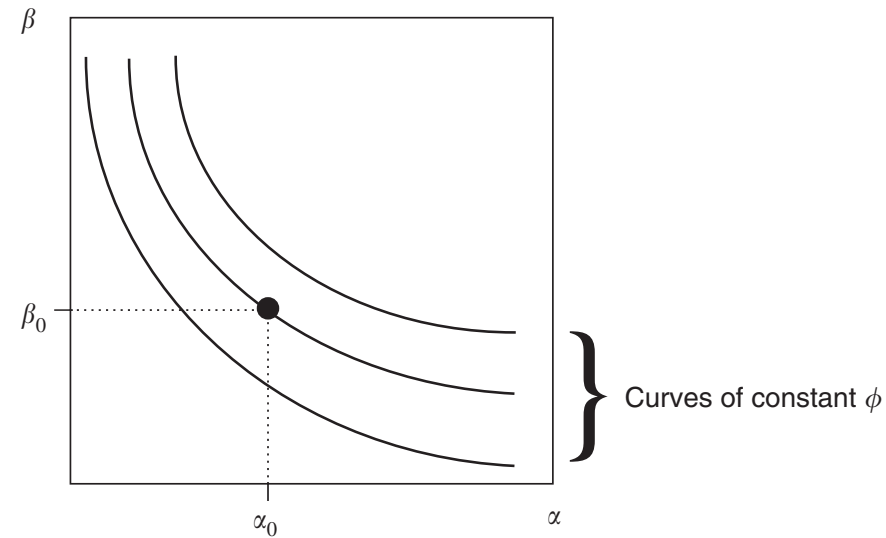

Fig. 6. Curves defined by $R\left(\alpha_{\mathrm{v}}, \beta_{\mathrm{v}} ; S^{*}(\alpha, \beta), I^{*}(\alpha, \beta)\right)=1$, or equivalently by $\phi\left(S^{*}(\alpha, \beta), I^{*}(\alpha, \beta)\right)=\phi_{0}$ with $\phi_{0}$ defined by $g\left(\alpha_{\mathrm{v}}, \beta_{\mathrm{v}}, \phi_{0}\right)=0$.

all types of individuals identically when it comes to determining the density-dependent deaths. If we proceed on this assumption, we may conclude that the environment of the infected individuals is at most two-dimensional, spanned by the densities of susceptible and infected individuals. What we still have to check is whether or not, by some quirk, the model components conspire around the subset of $\mathbf{R}^{4}$ defined by $R\left(\alpha_{\mathrm{v}}, \beta_{\mathrm{v}} ; S^{*}(\alpha, \beta)\right.$, $\left.I^{*}(\alpha, \beta)\right)=1$, to produce a lower effective dimension.

To prove that the effective environmental dimension for model (iv) is larger than 1, we first introduce a property that is shared by all evolutionary models having effective environmental dimension equal to 1 , and then show that model (iv) does not possess this property. Assume that there does exist a function $\phi: \mathbf{E} \rightarrow \mathbf{R}$ and a function $g: \mathbf{X} \times \mathbf{R} \rightarrow \mathbf{R}$, not necessarily increasing in its second argument, such that $\operatorname{sign} \ln \left(R\left(x_{\mathrm{v}} ; E_{\text {attr }} \quad(\mathrm{x})\right)=\operatorname{sign} \mathrm{g}\left(x_{\mathrm{v}}\right.\right.$; $\left.\phi\left(E_{\text {attr }}(x)\right)\right)$. Then the family of manifolds in $\mathbf{X}$ defined by $R\left(x_{\mathrm{v}} ; E_{\text {attr }}(x)\right)=1$, parameterized by $x_{\mathrm{v}}$, equals the family of manifolds defined by $\phi\left(E_{\text {attr }}(x)\right)=\phi_{0}$ with $\phi_{0}$ defined by $g\left(x_{\mathrm{v}} ; \phi_{0}\right)=0$, as is illustrated in Fig. 6. Translated into the notation of model (iv), this means that a curve through a point $(\alpha, \beta)=\left(\alpha_{0}, \beta_{0}\right)$ defined by $R\left(\alpha_{\mathrm{v}}, \beta_{\mathrm{v}} ; S^{*}(\alpha, \beta)\right.$, $\left.I^{*}(\alpha, \beta)\right)=1$ will not change if we change $\left(\alpha_{\mathrm{v}}, \beta_{\mathrm{v}}\right)$ in such a manner that the resulting curve still passes through the point $\left(\alpha_{0}, \beta_{0}\right)$. To check that this property does not hold for $R$ in (12), with $S^{*}$ and $I^{*}$ defined by (15), it suffices to calculate the derivative in $\left(\alpha_{0}, \beta_{0}\right)$ of different curves $R\left(\alpha_{\mathrm{v}}, \beta_{\mathrm{v}} ; S^{*}(\alpha, \beta), I^{*}(\alpha, \beta)\right)=1$, with $\left(\alpha_{\mathrm{v}}, \beta_{\mathrm{v}}\right) \in \mathbf{R}_{+}^{2}$, passing through $\left(\alpha_{0}, \beta_{0}\right)$, which is easily done through an implicit differentiation in the defining equation. Since this derivative depends on $\left(\alpha_{\mathrm{v}}, \beta_{\mathrm{v}}\right)$, the curves through $\left(\alpha_{0}, \beta_{0}\right)$ for different $\left(\alpha_{\mathrm{v}}, \beta_{\mathrm{v}}\right)$ do not coincide, as they should if the effective dimension of the environment equaled 1.

\section{Appendix B. Ascertaining continuous stability in more dimensions}

How can we resolve whether or not ESSs are evolutionarily attracting when mutational steps are not necessarily 
small, as is the assumption implicitly made in the consideration of IF-ESSs? Here we sketch, in a phrasing adapted to the latter problem, a research program that we expect to be helpful in addressing this open research question in the case in which the distributions of mutational steps are smooth and relatively wide.

We denote the potential variant trait combinations engendered by a heterozygote modifier as $x_{B}=\left(x_{B, a a}\right.$, $\left.x_{B . a A}, x_{B . a a}\right) \in \mathbf{X}^{3}$, with $\mathbf{X}$ denoting the space of traits of single individuals. A similar notation, $x_{b}=\left(x_{b, a a}, x_{b . a A}\right.$, $\left.x_{\text {b.aa }}\right) \in \mathbf{X}^{3}$, applies to the residents. We move the origin of the space $\mathbf{X}^{3}$ to the ESS. Moreover, we emphasize the dependence of $R_{\mathrm{B}}$ on the variant and resident traits, $R_{B}=R\left(x_{B} ; x_{b}\right)$. Using this notation, we define $H\left(x_{b}\right)=$ $\left\{x \mid R\left(x ; x_{b}\right)>1\right\}$, so that $H\left(x_{b}\right) \subset \mathbf{X}^{3}$ is the set of potentially successful invaders of $x_{b}$. It follows from Taylor-expanding $R$ that the family of sets $H\left(x_{b}\right)$ is scale-invariant close to the origin: if we neglect higher-order terms in $H\left(x_{b}\right)$, multiplying the space $\mathbf{X}^{3}$ with a constant will map the family onto itself. Fig. 7 illustrates such scale invariance for a simpler two-dimensional problem. Since, for small mutational steps, the invasion probability of a variant calculated from a branching-process approximation depends on $R$ linearly, this probability satisfies similar scale invariance. Moreover, by the smoothness and large extent of the mutation distribution, we may assume all variants arising from $X_{b}$ through mutation to be uniformly distributed in $H\left(x_{b}\right)$. For the time being, we assume that any successful invasion leads to a substitution. We can then decompose the process of sequential substitutions into an autonomous process on the unit sphere in $\mathbf{X}^{3}$, together with a subjugated radial process. The logarithm of the radial process is a

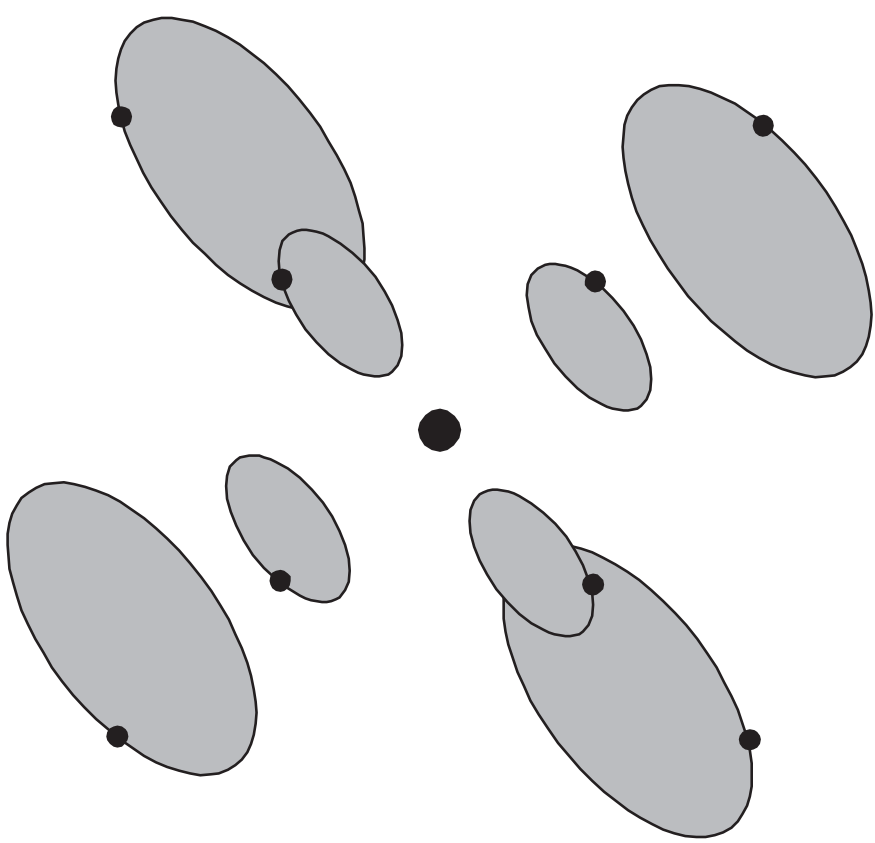

Fig. 7. Configuration of sets of variants with positive invasion fitness for different residents (small black dots) around an ESS (large black dot). random walk with dependent steps. Convergence or divergence of the radial process corresponds to convergence of the log-radial process to either $-\infty$ or $+\infty$. This convergence, in turn, depends on whether the steps are positive or negative on average. A negative average implies almost sure convergence of the radial process to zero, whereas a positive average implies that any neighborhood of zero will almost surely be left forever. Having said this, we run into the first real difficulty: to calculate these average steps, we first have to calculate the stationary distribution of the process on the unit sphere. Based on our work so far, we can only say that this distribution satisfies a forbidding looking integral equation.

A second difficulty is that invasion does not necessarily imply substitution. As we concentrate on only a small range of phenotypic possibilities, we may assume that the genotype-to-phenotype map is additive to first order of approximation. Close to the ESS, selection is weak relative to recombination. So, to the required order of approximation, we can describe a polymorphism in terms of the corresponding average phenotype $\bar{x}=\left(\bar{x}_{a a}, \bar{x}_{a A}, \bar{x}_{a a}\right)$, together with a list of variable length, corresponding to the number of modifiers, consisting of elements $\left(\pi_{B}, X_{B}\right)$, where $\pi_{B}=\left(p_{B}+q_{B}\right) / 2$ denotes the frequency on a modifier $B$, with $p_{B}$ being its frequency in the microgametes and $q_{B}$ its frequency in the macrogametes, and $x_{B}$ denotes the modifier's llelic effect. (Note that for given $\left(\pi_{B}, X_{B}\right), \pi_{b}$ and $x_{b}$ can be calculated from $\pi_{b}=1-\pi_{B}$ and $\pi_{b} x_{b}+$ $\pi_{B} x_{B}=0$. By writing $p_{B}=\pi_{B}+\delta_{B}$ and $q_{B}=\pi_{B}-\delta_{B}$ and by expanding the genetic recurrences around the IF-ESS, it can be seen, moreover, that up to second order $\pi_{B}$ satisfies a classical genetic equilibrium equation, with "viabilities" $v(x, E) / \bar{v}=[\tilde{\lambda}(x, E)+\tilde{\mu}(x, E)] / 2$ of a type considered by Zhivotovsky and Gavrilets (1993) and by Hermisson et al. (2003). Furthermore, $\delta_{B}$ is first order in the distance to the IF-ESS, and can, up to this order, be expressed explicitly in terms of the $\pi_{B}$ and the differences of the relative female and male gametic outputs, $\tilde{\mu}(x, E)-\tilde{\lambda}(x, E)$.) The states of the long-term evolutionary process then correspond to the population dynamically feasible lists of this kind. This full process satisfies the same scale invariance as before, and can therefore be decomposed into a scale-free configuration process, with states represented by $\bar{x} /\|\bar{x}\|$ together with lists $\left(\pi_{B}, x_{B} /\|\bar{x}\|\right)$, and a subjugated radial process $\|\bar{x}\|$. And, just as in the simple case, the log-radial process is a random walk. However, the task of calculating the stationary distribution of the configuration process, and from that the average step of the log-radial process, is even more daunting than for the earlier introduced toy process based on the (close to an ESS often incorrect) assumption that invasion implies substitution. The three reasons for yet providing this sketch are that (i) it indicates where the difficulties lie, (ii) it may put mathematicians on a potentially interesting track, and (iii) it shows that the local attractivity of an IF-ESS is an all-or-nothing phenomenon, and can thus be determined by a single simulation run. 
As a final point we remark that although the program sketched above is both mathematically interesting and possibly also rewarding, it is, for its biological meaning, predicated on the assumption that deterministic forces stay dominant. When the ESS is approached, the fitness values of invaders get closer and closer to zero, so that, for finite populations, the strength of selection will eventually become comparable to that of mutation and/or random drift. It thus depends on the interplay between many factors whether or not the conclusions derived from the idealization sketched above make biological sense. Salient questions are as follows. How close an approximation of the ESS are we interested in? What are the relative curvatures of the fitness landscape and of its dependence on the resident traits? What is the size of the population we are considering? What is the frequency of the occurrence of new modifier alleles with effects in the right range? How long has the process been going on since the last externally imposed change in the environment? Clarifying whether convergence occurs in the idealized case of unrestricted mutation limitation is thus only the natural first step in studying a diversity of factors and their interplay.

\section{References}

Anderson, R.M., May, R.M., 1982. Coevolution of hosts and parasites. Parasitology 85, 411-426.

Anderson, R.M., May, R.M., 1991. Infectious Diseases of Humans: Dynamics and Control. Oxford University Press, Oxford, UK.

Axelrod, R., Hamilton, W.D., 1981. The evolution of cooperation. Science 211, 1390-1396.

Bishop, D.T., Cannings, C., 1978. A generalized war of attrition. J. Theor. Biol. 70, 85-125.

Bodmer, W.F., 1965. Differential fertility in population genetics models. Genetics 51, 411-424.

Bulmer, M., 1994. Theoretical Evolutionary Ecology. Sinauer, Sunderland, MA, USA.

Calow, P., 1999. Encyclopedia of Ecology and Environmental Management. Blackwell Publishing, Oxford, UK.

Case, T.J., 1999. An Illustrated Guide to Theoretical Ecology. Oxford University Press, Oxford, UK.

Champagnat, N., Ferrière, N., Méléard, S. Unifying evolutionary dynamics: from individual stochastic processes to macroscopic models via timescale separation. Theor. Popul. Biol., this issue, doi:10.1016/ j.tpb.2005.10.004.

Charlesworth, B., 1976. Natural selection in age-structured population. In: Levin, S. (Ed.), Lectures in Mathematics in the Life Sciences, vol. 8. AMS, Providence, RI, pp. 69-78.

Charlesworth, B., 1994. Evolution in Age-Structured Populations, second ed. Cambridge University Press, Cambridge UK.

Cheptou, P.O., 2004. Allee effect and self-fertilization in hermaphrodites: reproductive assurance in demographically stable populations. Evolution 58, 2613-2621.

Christiansen, F.B., 1991. On conditions for evolutionary stability for a continuously varying character. Am. Nat. 138, 37-50.

Cressman, R., 1990. Evolutionarily stable strategies depending on population-density. Rocky Mountain J. Math. 20, 873-877.

Darwin, C., 1859. On the Origin of Species. John Murray, London, UK.

Dercole, F., Rinaldi, S., 2002. Evolution of cannibalistic traits: scenarios derived from adaptive dynamics. Theor. Popul. Biol. 62, 365-374.

Dercole, F., Ferrière, R., Rinaldi, S., 2002. Ecological bistability and evolutionary reversals under asymmetrical competition. Evolution 56, 1081-1090.
Diekmann, O., Heesterbeek, J.A.P., Metz, J.A.J., 1990. On the definition and the computation of the basic reproduction ratio $R_{0}$ in models for infectious diseases in heterogeneous populations. J. Math. Biol. 28, 365-382.

Diekmann, O., Gyllenberg, M., Huang, H., Kirkilionis, M., Metz, J.A.J., Thieme, H.R., 2001. On the formulation and analysis of general deterministic structured population models. II. Nonlinear Theory. J. Math. Biol. 43, 157-189.

Diekmann, O., Gyllenberg, M., Metz, J.A.J., 2003. Steady state analysis of structured population models. Theor. Popul. Biol. 63, 309-338.

Dieckmann, U., 2002. Adaptive dynamics of pathogen-host interactions. In: Dieckmann, U., Metz, J.A.J., Sabelis, M.W., Sigmund, K. (Eds.), Adaptive Dynamics of Infectious Diseases: In Pursuit of Virulence Management. Cambridge University Press, Cambridge, UK, pp. 39-59.

Dieckmann, U., Ferrière, R., 2004. Adaptive dynamics and evolving biodiversity. In: Ferrière, R., Dieckmann, U., Couvet, D. (Eds.), Evolutionary Conservation Biology. Cambridge University Press, Cambridge, UK, pp. 188-224.

Dieckmann, U., Law, R., 1996. The dynamical theory of coevolution: a derivation from stochastic ecological processes. J. Math. Biol. 34, 579-612.

Dieckmann, U., Metz, J.A.J., Doebeli, M., Tautz, D. (Eds.), 2004. Adaptive Speciation. Cambridge University Press, Cambridge, UK.

Doebeli, M., Dieckmann, U., 2000. Evolutionary branching and sympatric speciation caused by different types of ecological interactions. Am. Nat. 156, 77-101.

Durinx, M., Metz, J.A.J., 2005. Multi-type branching processes and adaptive dynamics of structured populations. In: Haccou, P., Jagers, P., Vatutin, V. (Eds.), Branching Processes in Biology: Variation, Growth and Extinction of Populations. Cambridge University Press, Cambridge, UK, pp. 266-277.

Ellner, S., 1996. Environmental fluctuations and the maintenance of genetic diversity in age- or stage-structured populations. Bull. Math. Biol. 58, 103-129.

Ernande, B., Dieckmann, U., Heino, M., 2002. Fisheries-induced changes in age and size at maturation and understanding the potential for selection-induced stock collapse. ICES CM 2002/Y:06.

Eshel, I., Feldman, M., 1984. Initial increase of new mutants and some continuity properties of ESS in two-locus systems. Am. Nat. 124, 631-640.

Eshel, I., Motro, U., Sansone, E., 1997. Continuous stability and evolutionary convergence. J. Theor. Biol. 185, 333-334.

Felsenstein, J., 1978-2005. Theoretical Evolutionary Genetics. Available online at http://evolution.gs.washington.edu/pgbook/pgbook.html.

Ferrière, R., 2000. Adaptive responses to environmental threats: evolutionary suicide, insurance, and rescue. Options Spring 2000, International Institute for Applied Systems Analysis, Laxenburg, Austria, pp. $12-16$.

Ferrière, R., Bronstein, J.L., Rinaldi, S., Gauduchon, M., Law, R., 2002. Cheating and the evolutionary stability of mutualism. Proc. R. Soc. London Ser. B 269, 773-780.

Fisher, R.A., 1930. The Genetical Theory of Natural Selection. Clarendon Press, Oxford, UK.

Fretwell, S.D., Lucas, H.J., 1970. On territorial behaviour influencing habitat distribution in birds. Acta Biotheor. 19, 16-36.

Geritz, S.A.H., Kisdi, É., Meszéna, G., Metz, J.A.J., 1998. Evolutionarily singular strategies and the adaptive growth and branching of the evolutionary tree. Evol. Ecol. 12, 35-57.

Geritz, S.A.H., Metz, J.A.J., Kisdi, E., Meszéna, G., 1997. Dynamics of adaptation and evolutionary branching. Phys. Rev. Lett. 78, 2024-2027.

Golubitsky, M., Schaeffer, D.G., 1985. Singularities and Groups in Bifurcation Theory, vol. 1, Applied Mathematical Sciences 51, Springer, Berlin, Heidelberg, New York, USA.

Guckenheimer, J., Holmes, P., 1997. Nonlinear Oscillations, Dynamical Systems, and Bifurcations of Vector Fields, third ed. Springer, New York, USA. 
Gyllenberg, M., Parvinen, K., 2001. Necessary and sufficient conditions for evolutionary suicide. Bull. Math. Biol. 63, 981-993.

Gyllenberg, M., Parvinen, K., Dieckmann, U., 2002. Evolutionary suicide and evolution of dispersal in structured metapopulations. J. Math. Biol. 45, 79-105.

Haldane, J.B.S., 1932. The Causes of Evolution. Harper, London, UK.

Hardin, G., 1968. The tragedy of the commons. Science 162, 1243-1248.

Hardy, G.H., 1908. Mendelian proportions in mixed populations. Science $28,49-50$.

Hermisson, J., Hansen, T.F., Wagner, G.P., 2003. Epistasis in polygenic traits and the evolution of genetic architecture under stabilizing selection. Am. Nat. 161, 708-734.

Heino, M., Metz, J.A.J., Kaitala, V., 1997. Evolution of mixed maturation strategies in semelparous life-histories: the crucial role of dimensionality of feedback environment. Phil. Trans. R. Soc. Lond. Ser. B 352, $1647-1655$.

Heino, M., Metz, J.A.J., Kaitala, V., 1998. The enigma of frequentlydependent selection. Trends Ecol. Evol. 13, 367-370.

Hofbauer, J., Sigmund, K., 1998. Evolutionary Games and Population Dynamics. Cambridge University Press, Cambridge, UK.

Karlin, S., Lessard, S., 1986. Theoretical Studies on Sex Ratio Evolution, Monographs in Population Biology, vol. 22. Princeton University Press, Princeton, NJ, USA.

Kirkpatrick, M., 1996. Genes and adaptation: a pocket guide to theory. In: Rose, M.R., Lauder, G.V. (Eds.), Adaptation. Academic Press, San Diego, USA, pp. 125-128.

Kokko, H., Brooks, R., 2003. Sexy to die for? Sexual selection and the risk of extinction. Ann. Zool. Fennici 40, 207-219.

Le Galliard, J.F., Ferrière, R., Dieckmann, U., 2003. The adaptive dynamics of altruism in spatially heterogeneous populations. Evolution $57,1-17$.

Leimar, O., 2001. Evolutionary change and Darwinian demons. Selection 2, 65-72.

Leimar, O., 2005. The evolution of phenotypic polymorphism: randomized strategies versus evolutionary branching. Am. Nat. 165, 669-681.

Leimar, O. Multidimensional convergence stability and the canonical adaptive dynamics. In: Dieckmann, U., Metz, J.A.J. (Eds.), Elements of Adaptive Dynamics. Cambridge University Press, Cambridge, UK, in press.

Levins, R., 1962. Theory of fitness in a heterogeneous environment. I. The fitness set and adaptive function. Am. Nat. 96, 361-373.

Levins, R., 1968. Evolution in Changing Environments. Princeton University Press, Princeton, NJ, USA.

Lewontin, R.C., 1958. A general method for investigating the equilibrium of gene frequencies in a population. Genetics 43, 419-434.

Liberman, U., 1988. External stability and ESS: criteria for the initial increase of a new mutant allele. J. Math. Biol. 26, 477-485.

Mandel, S.P.H., 1971. Owen's model of a genetical system with differential viability between sexes. Heredity 26, 49-63.

Mathias, A., Kisdi, É. Evolutionary branching and coexistence of germination strategies. In: Dieckmann, U., Metz, J.A.J. (Eds.), Elements of Adaptive Dynamics. Cambridge University Press, Cambridge, UK, in press.

Matsuda, H., Abrams, P.A., 1994a. Runaway evolution to self-extinction under asymmetrical competition. Evolution 48, 1764-1772.

Matsuda, H., Abrams, P.A., 1994b. Timid consumers - self-extinction due to adaptive change in foraging and anti-predator effort. Theor. Popul. Biol. 45, 76-91.

Maynard Smith, J., 1978. The Evolution of Sex. Cambridge University Press, Cambridge, UK.

Maynard Smith, J., 1982. Evolution and the Theory of Games. Cambridge University Press, Cambridge, UK.

Maynard Smith, J., Price, G.R., 1973. The logic of animal conflict. Nature 246, 15-18.
Metz, J.A.J., de Roos, A.M., 1992. The role of physiologically structured population models within a general individual-based modeling perspective. In: DeAngelis, D.L., Gross, L.J. (Eds.), Individual-based Models and Approaches in Ecology. Routledge, Chapman \& Hall, New York, USA, pp. 88-111.

Metz, J.A.J., Diekmann, O., 1986. The Dynamics of Physiologically Structured Populations. Springer Lecture Notes in Biomathematics, vol. 68. Springer, Berlin, Germany.

Metz, J.A.J., Geritz, S.A.H., Meszéna, G., Jacobs, F.J.A., van Heerwaarden, J.S., 1996a. Adaptive dynamics, a geometrical study of the consequences of nearly faithful reproduction. In: van Strien, S.J., Verduyn Lunel, S.M. (Eds.), Stochastic and Spatial Structures of Dynamical Systems. North-Holland, Amsterdam, The Netherlands, pp. 183-231.

Metz, J.A.J., Mylius, S.D., Diekmann, O., 1996b. When does evolution optimise? On the relation between types of tensity dependence and evolutionarily stable life history parameters. IIASA Working Paper WP-96-04. Available online at http://www.iiasa.ac.at/cgi-bin/ pubsrch?WP96004.

Metz, J.A.J., Nisbet, R.M., Geritz, S.A.H., 1992. How should we define fitness for general ecological scenarios. Trends Ecol. Evol. 7, 198-202.

Mylius, S.D., Diekmann, O., 1995. On evolutionarily stable life histories, optimization and the need to be specific about density dependence. Oikos 74, 218-224.

Owen, A.R.G., 1952. A genetical system admitting of two distinct stable equilibria under natural selection. Heredity 7, 97-102.

Parvinen, K., 2006. Evolutionary suicide. Acta Biotheor. 53, 241-264.

Rand, D.A., Wilson, H.B., McGlade, J.M., 1994. Dynamics and evolution: evolutionarily stable attractors, invasion exponents and phenotypic dynamics. Phil. Trans. R. Soc. Lond. Ser. B 343, 261-283.

Roff, D.A., 1992. The Evolution of Life Histories: Theory and Analysis. Chapman \& Hall, New York, USA.

Roughgarden, J., 1971. Density-dependent natural selection. Ecology 52, 453-468.

Roughgarden, J., 1979. Theory of Population Genetics and Evolutionary Ecology: An Introduction. Macmillan, New York, USA.

Schuster, P., Sigmund, K., 1983. Replicator dynamics. J. Theor. Biol. 100, 533-538.

Stearns, S.C., 1992. The Evolution of Life Histories. Oxford University Press, Oxford, UK.

Taylor, P.D., 1989. Evolutionary stability in one-parameter models under weak selection. Theor. Popul. Biol. 36, 125-143.

Taylor, P.D., Jonker, L., 1978. Evolutionarily stable strategies and game dynamics. Math. Biosci. 40, 145-156.

Webb, C.T., 2003. A complete classification of Darwinian extinction in ecological interactions. Am. Nat. 161, 181-205.

Weinberg, W., 1908. Über den Nachweis der Vererbung beim Menschen. Jahresh. Ver. Vaterl. Naturk. Württemberg 64, 368-382.

Williams, G.C., 1966. Adaptation and Natural Selection: A Critique of Some Current Evolutionary Thought. Princeton University Press, Princeton USA.

Wright, S., 1932. The roles of mutation, inbreeding, crossbreeding and selection in evolution. Proceedings of the 6th International Congress of Genetics 1, 356-366.

Wright, S., 1967. Surfaces of selective value. Proc. Nat. Acad. Sci. USA $102,81-84$.

Wynne-Edwards, V.C., 1962. Animal Dispersion in Relation to Social Behaviour. Oliver and Boyd, Edinburgh, UK.

Yodzis, P., 1989. Introduction to Theoretical Ecology. Harper and Row, New York, USA.

Zhivotovsky, L.A., Gavrilets, S., 1993. Quantitative variability and multilocus polymorphism under epistatic selection. Theor. Popul. Biol. 42, 254-283. 